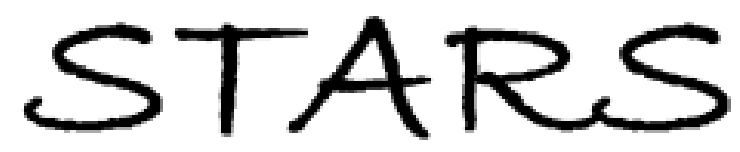

University of Central Florida

STARS

$1-1-2010$

\title{
Effects of Heat Transfer on the Stagnation Flow of a Third-Order Fluid over a Shrinking Sheet
}

\author{
Sohail Nadeem \\ Anwar Hussain \\ Kuppalapalle Vajravelu \\ University of Central Florida
}

Find similar works at: https://stars.library.ucf.edu/facultybib2010 University of Central Florida Libraries http://library.ucf.edu

This Article is brought to you for free and open access by the Faculty Bibliography at STARS. It has been accepted for inclusion in Faculty Bibliography 2010 s by an authorized administrator of STARS. For more information, please contact STARS@ucf.edu.

\section{Recommended Citation}

Nadeem, Sohail; Hussain, Anwar; and Vajravelu, Kuppalapalle, "Effects of Heat Transfer on the Stagnation Flow of a Third-Order Fluid over a Shrinking Sheet" (2010). Faculty Bibliography 2010s. 577.

https://stars.library.ucf.edu/facultybib2010/577

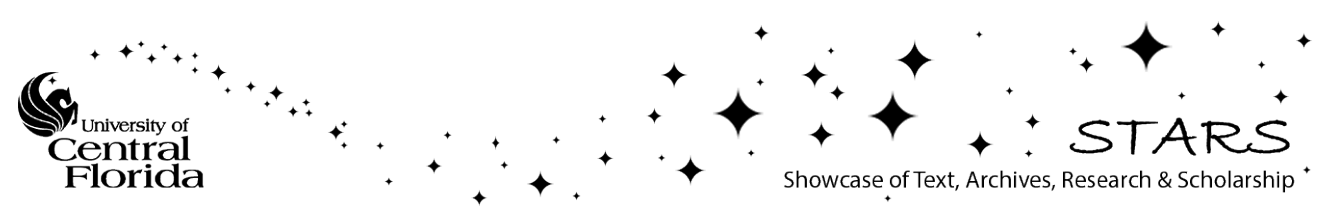




\title{
Effects of Heat Transfer on the Stagnation Flow of a Third-Order Fluid over a Shrinking Sheet
}

\author{
Sohail Nadeem ${ }^{\mathrm{a}}$, Anwar Hussain ${ }^{\mathrm{a}}$, and Kuppalapalle Vajravelu ${ }^{\mathrm{b}}$ \\ ${ }^{\text {a }}$ Department of Mathematics, Quaid-i-Azam University, 45320 Islamabad, Pakistan \\ b Department of Mathematics, University of Central Florida, Orlando, Fl 32816, USA \\ Reprint requests to S. N.; E-mail: snqau@ hotmail.com
}

Z. Naturforsch. 65a, 969 - 994 (2010); received August 3, 2009 / revised January 11, 2010

\begin{abstract}
This paper is devoted to the study of a stagnation point flow of an incompressible third-order fluid towards a shrinking sheet (with heat transfer). The governing nonlinear partial differential equations are reduced into nonlinear ordinary differential equations by means of a similarity transformation and then solved by the homotopy analysis method. Two types of flow problems, namely, (i) two dimensional stagnation flow toward a shrinking sheet and (ii) axisymmetric stagnation flow towards an axisymmetric shrinking surface have been discussed. Also, two types of boundary conditions are taken into account: (i) prescribed surface temperature (PST) and (ii) prescribed heat flux (PHF) case. The effects of various emerging parameters of non-Newtonian fluid have been investigated in detail and shown pictorically. The convergence of the solutions have been discussed through $\hbar$-curves and residual error. For further validity, the homotopy Padé approximation is also applied.
\end{abstract}

Key words: Stagnation Flow; Heat Transfer; Third-Order Fluid; Shrinking Sheet;

Homotopy Analysis Method.

\section{Introduction}

The effect of heat transfer over a stretching surface has been extensively investigated by many researchers during the last few years because of its applications in industry and technology. Such applications include heat treatment of materials manufactured in an extrusion process and a casting process of materials etc. After the first investigation of Sakiadis [1] numerous researchers have discussed boundary layer flow caused by a stretching surface [2-5]. Recently, El-Aziz [6] has discussed the effects of temperature dependent viscosity and thermal conductivity on a three-dimensional flow over a stretching surface. The influence of thermal radiation on the boundary layer flow due to an exponentially stretching surface have been examined by Sajid and Hayat [7]. Liu and Anderson [8] have discussed the heat transfer in a liquid film on an unsteady stretching sheet. The boundary layer flow and heat transfer over an exponential stretching sheet for a viscoelastic (second-grade) fluid have been studied by Khan and Sanjayanand [9]. Elbashbeshy and Bazid [10] have discussed the heat transfer over an unsteady stretching surface with internal heat generation or absorption. The heat and mass transfer in the boundary layers on an exponentially stretching con- tinuous surface have been examined by Magyari and Keller [11]. Zakaria [12] has studied magnetohydrodynamic viscoelastic boundary layer flow past a stretching plate and heat transfer. Heat transfer in a porous medium over a stretching surface with internal heat generation and suction or injection have been studied by Elpashbeshy and Bazid [13]. The boundary layer flow due to stagnation flows and a stretching surface is another rich area which has a number of industrial and engineering applications: fluid in rotating blades, cooling of silicon wafers, extrusion of a polymer in a melt spinning process, and the extrudate from the die is generally drawn and simultaneously stretched into a sheet which is then solidified through quenching or gradual cooling by direct contact with water.

In view of these Mahapatra and Gupta [14] have examined the magnetohydrodynamic (MHD) stagnation point flow towards a stretching surface. The unsteady boundary layer flow in the region of the stagnation point on a stretching surface has been discussed by Nazar et al. [15]. Lok et al. [16] examined the mixed convection flow of a micropolar fluid (near the stagnation point) on a vertical surface. The mixed convection flow near a non-orthogonal stagnation point towards a stretching vertical plate has been studied by Yian et al. [17]. 
In the references cited above, the boundary layer flow due to a stretching surface has been studied. There is another area which has attracted the attention of researchers due to its applications known as shrinking sheet phenomena. A process in which the velocity on the sheet towards a fixed point is known as shrinking phenomena. Only a limited attention have been given to this rich area. Mentioned may be the works of [1822]. Recently, Wang [23] has discussed the stagnation flow towards a shrinking sheet. Motivated by the above analysis, the aim of the present paper is to discuss the stagnation flow of a third-grade fluid towards a shrinking sheet with heat transfer analysis. Only few papers dealing with the flow of a third-grade fluid towards a stretching sheet have been reported [24-25]. To the best knowledge of the authors no attempt has been made to discuss the third-grade fluid towards a shrinking sheet. The governing equations of motion and energy are simplified by using suitable similarity transformations at two types of problems and discussed namely (i) two dimensional stagnation flow towards a shrinking sheet and (ii) on axisymmetric stagnation flow of a third-grade fluid towards an axially symmetric shrinking sheet. The reduced problems are then solved analytically (semi numerically) by the homotopy analysis method (HAM). Some recent developments on the method are given in [26-33]. The physical features of the various parameters have been discussed through graphs. The convergence of HAM solutions have been checked through $\hbar$-curves and residual error.

\section{Formulation of the Problem}

Consider an incompressible two-dimensional stagnation flow of a third-order fluid towards a shrinking sheet. The velocity components along $x, y$, and $z$ axis are denoted by $u, v$, and $w$, respectively. The potential stagnation flow at infinity is taken as $u=a x$, $w=-a z$, whereas stretching (shrinking) surface, the velocity components are considered as $u=b(x+c)$, $w=0$. Here $b$ is the stretching rate (shrinking if $b<0$ ) and $-c$ is the location of the stretching origin. The flow and heat characteristics are governed by the following boundary layer equations:

$$
\begin{aligned}
& \frac{\partial u}{\partial x}+\frac{\partial v}{\partial y}+\frac{\partial w}{\partial z}=0, \\
& u \frac{\partial u}{\partial x}+w \frac{\partial u}{\partial z}=-\frac{1}{\rho} \frac{\partial p}{\partial x}+v \frac{\partial^{2} u}{\partial z^{2}}
\end{aligned}
$$

$$
\begin{aligned}
& +\frac{\alpha_{1}}{\rho}\left(u \frac{\partial^{3} u}{\partial x \partial z^{2}}+\frac{\partial u}{\partial x} \frac{\partial^{2} u}{\partial z^{2}}+3 \frac{\partial u}{\partial z} \frac{\partial^{2} u}{\partial x \partial z}+w \frac{\partial^{3} u}{\partial z^{3}}\right) \\
& +2 \frac{\alpha_{2}}{\rho} \frac{\partial u}{\partial z} \frac{\partial^{2} u}{\partial x \partial z}+6 \frac{\left(\beta_{2}+\beta_{3}\right)}{\rho}\left(\frac{\partial u}{\partial z}\right)^{2} \frac{\partial^{2} u}{\partial z^{2}} \\
& \rho C_{\mathrm{p}}\left(u \frac{\partial T}{\partial x}+w \frac{\partial T}{\partial z}\right)=k \frac{\partial^{2} T}{\partial z^{2}}+\mu\left(\frac{\partial u}{\partial z}\right)^{2} \\
& +\alpha_{1}\left(u \frac{\partial u}{\partial z} \frac{\partial^{2} u}{\partial x \partial z}+w \frac{\partial u}{\partial z} \frac{\partial^{2} u}{\partial z^{2}}\right)+2\left(\beta_{2}+\beta_{3}\right)\left(\frac{\partial u}{\partial z}\right)^{4}
\end{aligned}
$$

where $v=\left(\frac{\mu}{\rho}\right)$ is the kinematic viscosity, $\rho$ is the density, $\alpha_{1}, \alpha_{2}$, and $\beta_{3}$ are material constants of the thirdorder fluid, $T$ is the temperature, $C_{\mathrm{p}}$ the specific heat at constant pressure, and $k$ is the thermal conductivity. Note that $v$ and $\frac{\alpha_{i}}{\rho}(i=1,2)$ being $O\left(\delta^{2}\right), \frac{\beta_{i}}{\rho}(i=1$, $2,3)$ being $O\left(\delta^{4}\right)$, and terms of $O(\delta)$ or higher are neglected (where $\delta$ being the thickness of the boundary layer).

\section{Two-Dimensional Stagnation Flow}

Defining [23]

$$
\begin{aligned}
& \eta=\sqrt{\frac{a}{v}} z, \quad u=a x f^{\prime}(\eta)+b c h(\eta), \\
& v=0, \quad w=-\sqrt{a v} f(\eta) .
\end{aligned}
$$

Then (1) is satisfied identically and (2) yields

$$
\begin{aligned}
& f^{\prime \prime \prime}+f f^{\prime \prime}-f^{\prime 2}+1+\beta\left(2 f^{\prime} f^{\prime \prime \prime}+3 f^{\prime \prime 2}-f f^{I V}\right) \\
& +2 A f^{\prime \prime 2}+6 B S f^{\prime \prime 2} f^{\prime \prime \prime}+6 B Z f^{\prime \prime 2} h^{\prime \prime}+6 B K f^{\prime \prime \prime} h^{2} \\
& +12 B Z h^{\prime} f^{\prime \prime} f^{\prime \prime \prime}+12 B K h^{\prime} f^{\prime \prime} h^{\prime \prime}=0 \\
& h^{\prime \prime}+f h^{\prime}-f^{\prime} h+\beta\left(h f^{\prime \prime \prime}+f^{\prime} h^{\prime \prime}+3 f^{\prime \prime} h^{\prime}-f h^{\prime \prime \prime}\right) \\
& +2 A f^{\prime \prime} h^{\prime}+6 B K h^{\prime 2} h^{\prime \prime}=0,
\end{aligned}
$$

where $\beta=\frac{\alpha_{1} a}{\rho v}, A=\frac{\alpha_{2} a}{\rho v}, B=\frac{a^{2}\left(\beta_{2}+\beta_{3}\right)}{\rho v}, K=\frac{b^{2} c^{2}}{a v}$, $S=\frac{a x^{2}}{v}$, and $Z=\frac{b c x}{v}$ are the non-dimensional fluid parameters. The associated boundary conditions are

$$
\begin{array}{ll}
f(0)=0, & f^{\prime}(0)=b / a=\alpha, \quad f^{\prime}(\infty)=1, \\
h(0)=1, & h(\infty)=0,
\end{array}
$$

where a prime denotes the derivative with respect to $\eta$.

\subsection{The Prescribed Surface Temperature (PST) Case}

Here

$$
\begin{aligned}
& T=T_{\mathrm{w}}=T_{\infty}+A\left(\frac{x}{l}\right)^{2} \text { at } z=0, \\
& T \rightarrow T_{\infty} \text { as } z \rightarrow \infty,
\end{aligned}
$$


where $A$ is a constant. Defining

$$
\theta(x, \eta)=\frac{T-T_{\infty}}{T_{w}-T_{\infty}} .
$$

Using (8) and (9), then (3) yields

$$
\begin{aligned}
& \theta^{\prime \prime}+P_{\mathrm{r}}\left(f \theta^{\prime}-2 f^{\prime} \theta-x f^{\prime} \frac{\partial \theta}{\partial x}-2 M h \theta-M x h \frac{\partial \theta}{\partial x}\right) \\
& +P_{\mathrm{r}} E\left(f^{\prime \prime 2}+2 M f^{\prime \prime} h^{\prime}\right)+\beta P_{\mathrm{r}} E\left(f^{\prime} f^{\prime \prime 2}+M f^{\prime} f^{\prime \prime} h^{\prime}\right. \\
& \left.+M h f^{\prime \prime 2}-f f^{\prime \prime} f^{\prime \prime \prime}-M f f^{\prime \prime} h^{\prime \prime}-M f h^{\prime} f^{\prime \prime \prime}\right)+2 P_{\mathrm{r}} E B S \\
& \cdot\left(f^{\prime \prime 4}+4 M f^{\prime \prime 3} h^{\prime}+6 M^{2} f^{\prime \prime 2} h^{\prime 2}+4 M^{3} f^{\prime \prime} h^{\prime 3}\right)=0, \quad(10) \\
& h^{\prime 2}+\beta\left(h h^{\prime} f^{\prime \prime}-f h^{\prime} h^{\prime \prime}\right)+2 B S M^{2} h^{\prime 4}=0 .
\end{aligned}
$$

The corresponding boundary conditions become

$$
\theta=1 \text { at } \eta=0 \text { and } \theta \rightarrow 0 \text { as } \eta \rightarrow \infty \text {. }
$$

In the above equations $P_{\mathrm{r}}=\frac{\mu C_{\mathrm{p}}}{k}, E=\frac{a^{2} l^{2}}{C_{\mathrm{p}} A}$ are the Prandtl number and Eckert number, respectively, while $M=\frac{b c}{a x}$.

\subsection{The Prescribed Surface Heat Flux (PHF) Case} form:

Here the boundary conditions are of the following

$$
\begin{aligned}
& -k \frac{\partial T}{\partial z}=q_{\mathrm{w}}=D\left(\frac{x}{l}\right)^{2} \text { at } z=0, \\
& T \rightarrow T_{\infty} \text { as } z \rightarrow \infty
\end{aligned}
$$

Taking

$$
T-T_{\infty}=\frac{D}{k}\left(\frac{x}{l}\right)^{2} \sqrt{\frac{\nu}{a}} \varphi(x, \eta) .
$$

Making use of (14), then (3) along with its boundary conditions (13) take the following form:

$$
\begin{aligned}
& \varphi^{\prime}(x, \eta)=-1 \text { at } \eta=0, \text { and } \\
& \varphi(x, \eta)=0 \text { as } \eta \rightarrow \infty .
\end{aligned}
$$

The governing energy equation is the same as defined in the previous section except the value of $E$ is different which is given by

$$
E=\frac{k a^{2} l^{2}}{D C_{\mathrm{p}}} \sqrt{\frac{a}{v}} .
$$

\section{Axisymmetric Stagnation Flow Towards a Shrinking Surface}

Wang [23] considered in his analysis the axisymmetric case; it is not necessary that one should take cylinderical coordinates. It is possible to achieve the same equations in Cartesian coordinates by taking

$$
\begin{aligned}
& \eta(x, z)=\sqrt{\frac{a}{v}} z, \quad u=a x g^{\prime}(\eta)+b c n(\eta), \\
& v=a y g^{\prime}(\eta), \quad w=-2 \sqrt{a v} g(\eta) .
\end{aligned}
$$

With the help of (17), (1) is identically satisfied and (2) takes the following form:

$$
\begin{aligned}
& g^{\prime \prime \prime}+2 g g^{\prime \prime}-g^{2}+1+\beta\left(2 g^{\prime} g^{\prime \prime \prime}+3 g^{\prime \prime 2}-2 g g^{I V}\right) \\
& +2 A g^{\prime \prime 2}+6 B S g^{\prime \prime 2} g^{\prime \prime \prime}+6 B Z g^{\prime \prime 2} n^{\prime \prime}+6 B K g^{\prime \prime \prime} n^{\prime 2} \\
& +12 B Z n^{\prime} g^{\prime \prime} g^{\prime \prime \prime}+12 B K n^{\prime} g^{\prime \prime} n^{\prime \prime}=0
\end{aligned}
$$

$$
\begin{aligned}
& n^{\prime \prime}+2 g n^{\prime}-g^{\prime} n+\beta\left(n g^{\prime \prime \prime}+g^{\prime} n^{\prime \prime}+3 g^{\prime \prime} n^{\prime}-2 g n^{\prime \prime \prime}\right) \\
& +2 A g^{\prime \prime} n^{\prime}+6 B K n^{\prime 2} n^{\prime \prime}=0 .
\end{aligned}
$$

The relevant boundary conditions are

$$
\begin{array}{ll}
g(0)=0, & g^{\prime}(0)=b / a=\alpha, \quad g^{\prime}(\infty)=1, \\
n(0)=1, & n(\infty)=0 .
\end{array}
$$

\subsection{The Prescribed Surface Temperature (PST) Case}

Here

$$
\begin{aligned}
& T=T_{\mathrm{w}}=T_{\infty}+A\left(\frac{x}{l}\right)^{2} \text { at } z=0, \\
& T \rightarrow T_{\infty} \text { as } z \rightarrow \infty
\end{aligned}
$$

where $A$ is a constant. Defining

$$
\phi(x, \eta)=\frac{T-T_{\infty}}{T_{\mathrm{w}}-T_{\infty}} .
$$

Using (8) and (9), then (3) yields

$$
\begin{aligned}
& \phi^{\prime \prime}+P_{\mathrm{r}}\left(2 g \phi^{\prime}-2 g^{\prime} \phi-x g^{\prime} \frac{\partial \phi}{\partial x}-2 M n \phi-M x n \frac{\partial \phi}{\partial x}\right) \\
& +P_{\mathrm{r}} E\left(g^{\prime \prime 2}+2 M g^{\prime \prime} n^{\prime}\right)+P_{\mathrm{r}} E \beta\left(g^{\prime} g^{\prime \prime 2}+M g^{\prime} g^{\prime \prime} n^{\prime}\right. \\
& \left.+M n g^{\prime \prime 2}-2 g g^{\prime \prime} g^{\prime \prime \prime}-2 M g g^{\prime \prime} n^{\prime \prime}-2 M g n^{\prime} g^{\prime \prime \prime}\right) \\
& +2 P_{\mathrm{r}} E B S\left(g^{\prime \prime 4}+4 M g^{\prime \prime 3} n^{\prime}+6 M^{2} g^{\prime \prime 2} n^{\prime 2}\right. \\
& \left.+4 M^{3} g^{\prime \prime} n^{\prime 3}\right)=0 \\
& \quad n^{\prime 2}+\beta\left(n n^{\prime} g^{\prime \prime}-g n^{\prime} n^{\prime \prime}\right)+2 B S M^{2} n^{\prime 4}=0
\end{aligned}
$$

The corresponding boundary conditions are

$$
\phi=1 \text { at } \eta=0 \text { and } \phi \rightarrow 0 \text { as } \eta \rightarrow \infty,
$$


where $P_{\mathrm{r}}=\frac{\mu C_{\mathrm{p}}}{k}, E=\frac{a^{2} l^{2}}{C_{\mathrm{p}} A}$ are the Prandtl number and the Eckert number, respectively, while $M=\frac{b c}{a x}$.

\subsection{The Prescribed Surface Heat Flux (PHF) Case}

Here the boundary conditions are of the following form:

$$
\begin{aligned}
& -k \frac{\partial T}{\partial z}=q_{\mathrm{w}}=D\left(\frac{x}{l}\right)^{2} \text { at } z=0, \\
& T \rightarrow T_{\infty} \text { as } z \rightarrow \infty
\end{aligned}
$$

Taking

$$
T-T_{\infty}=\frac{D}{k}\left(\frac{x}{l}\right)^{2} \sqrt{\frac{v}{a}} \psi(x, \eta),
$$

we obtain the differential equations (23) and (24) with the following boundary conditions:

$$
\begin{aligned}
& \psi^{\prime}(x, \eta)=-1, \text { at } \eta=0, \text { and } \\
& \psi(x, \eta)=0 \text { as } \eta \rightarrow \infty .
\end{aligned}
$$

In this case the Eckert number is defined as

$$
E=\frac{k a^{2} l^{2}}{D C_{\mathrm{p}}} \sqrt{\frac{a}{v}}
$$

\section{Solution of the Problem}

\subsection{Solution for Two-Dimensional Stagnation Flow}

Towards a Shrinking Sheet

For HAM solution, we select the following initial approximations and auxiliary linear operators:

$$
\begin{aligned}
& f_{01}(\eta)=(1-\alpha)(\exp (-\eta)-1)+\eta, \\
& h_{01}(\eta)=\exp (-\eta), \\
& \theta_{01}(\eta)=\exp (-\eta), \\
& \mathcal{L}_{01}[\widehat{f}(\eta ; p)]=\frac{\partial^{3} \widehat{f}(\eta ; p)}{\partial \eta^{3}}+\frac{\partial^{2} \widehat{f}(\eta ; p)}{\partial \eta^{2}}, \\
& \mathcal{L}_{02}[\widehat{h}(\eta ; p)]=\frac{\partial^{2} \widehat{h}(\eta ; p)}{\partial \eta^{2}}+\frac{\partial \widehat{h}(\eta ; p)}{\partial \eta}, \\
& \mathcal{L}_{03}[\widehat{\theta}(\eta ; p)]=\frac{\partial^{2} \widehat{\theta}(\eta ; p)}{\partial \eta^{2}}+\frac{\partial \widehat{\theta}(\eta ; p)}{\partial \eta},
\end{aligned}
$$

where the (subscript 01 means the first-order and the other subscripts denotes the next-orders) and the properties satisfied by the auxiliary linear operator are

$$
\begin{aligned}
& \mathcal{L}_{01}\left[C_{1}+C_{2} \eta+C_{3} \mathrm{e}^{-\eta}\right]=0, \\
& \mathcal{L}_{02}\left[C_{4}+C_{5} \mathrm{e}^{-\eta}\right]=0, \\
& \mathcal{L}_{03}\left[C_{6}+C_{7} \mathrm{e}^{-\eta}\right]=0,
\end{aligned}
$$

in which $C_{i}(i=1-7)$ are arbitrary constants. If $p \in$ $[0,1]$ is an embedding parameter and $\hbar_{i}(i=1-4)$ are non-zero auxiliary parameters, then the zeroth-order and $m$ th-order deformation problems are as follows:

\subsubsection{Zeroth-Order Deformation Problems:}

$$
\begin{aligned}
& (1-p) \mathcal{L}_{01}\left[\widehat{f}(\eta ; p)-f_{01}(\eta)\right] \\
& =p \hbar_{1} \mathcal{N}_{1}[\widehat{f}(\eta ; p)] \text {, } \\
& (1-p) \mathcal{L}_{02}\left[\widehat{h}(\eta ; p)-h_{01}(\eta)\right] \\
& =p \hbar_{2} \mathcal{N}_{2}[\widehat{h}(\eta ; p), \widehat{f}(\eta ; p)], \\
& (1-p) \mathcal{L}_{03}\left[\widehat{\theta}(\eta ; p)-\theta_{01}(\eta)\right] \\
& =p \hbar_{3} \mathcal{N}_{3}[\widehat{\theta}(\eta ; p), \widehat{f}(\eta ; p), \widehat{h}(\eta ; p)], \\
& \widehat{f}(0 ; p)=0, \quad \widehat{f}^{\prime}(0 ; p)=\alpha, \quad \widehat{f}^{\prime}(\infty ; p)=1, \\
& \widehat{h}(0 ; p)=1, \quad \widehat{h}(\infty ; p)=0, \\
& \widehat{\boldsymbol{\theta}}(0 ; p)=1, \quad \widehat{\boldsymbol{\theta}}(\infty ; p)=0, \\
& \mathcal{N}_{1}[\widehat{f}(\eta ; p)]=\frac{\partial^{3} \widehat{f}(\eta ; p)}{\partial \eta^{3}}+\widehat{f}(\eta ; p) \frac{\partial^{2} \widehat{f}(\eta ; p)}{\partial \eta^{2}} \\
& -\left(\frac{\partial \widehat{f}(\eta ; p)}{\partial \eta}\right)^{2}+1+\beta\left[2 \frac{\partial \widehat{f}(\eta ; p)}{\partial \eta} \frac{\partial^{3} \widehat{f}(\eta ; p)}{\partial \eta^{3}}\right. \\
& \left.+3\left(\frac{\partial^{2} \widehat{f}(\eta ; p)}{\partial \eta^{2}}\right)^{2}-\widehat{f}(\eta ; p) \frac{\partial^{4} \widehat{f}(\eta ; p)}{\partial \eta^{4}}\right] \\
& +2 A\left(\frac{\partial^{2} \widehat{f}(\eta ; p)}{\partial \eta^{2}}\right)^{2}+6 B S\left(\frac{\partial^{2} \widehat{f}(\eta ; p)}{\partial \eta^{2}}\right)^{2} \frac{\partial^{3} \widehat{f}(\eta ; p)}{\partial \eta^{3}} \\
& +6 B Z\left(\frac{\partial^{2} \widehat{f}(\eta ; p)}{\partial \eta^{2}}\right)^{2} \frac{\partial^{2} \widehat{h}(\eta ; p)}{\partial \eta^{2}} \\
& +6 B K \frac{\partial^{3} \widehat{f}(\eta ; p)}{\partial \eta^{3}}\left(\frac{\partial \widehat{h}(\eta ; p)}{\partial \eta}\right)^{2} \\
& +12 B Z \frac{\partial \widehat{h}(\eta ; p)}{\partial \eta} \frac{\partial^{2} \widehat{f}(\eta ; p)}{\partial \eta^{2}} \frac{\partial^{3} \widehat{f}(\eta ; p)}{\partial \eta^{3}} \\
& +12 B K \frac{\partial \widehat{h}(\eta ; p)}{\partial \eta} \frac{\partial^{2} \widehat{f}(\eta ; p)}{\partial \eta^{2}} \frac{\partial^{2} \widehat{h}(\eta ; p)}{\partial \eta^{2}},
\end{aligned}
$$


$\mathcal{N}_{2}[\widehat{f}(\eta ; p), \widehat{h}(\eta ; p)]=\frac{\partial^{2} \widehat{h}(\eta ; p)}{\partial \eta^{2}}+\widehat{f}(\eta ; p) \frac{\partial \widehat{h}(\eta ; p)}{\partial \eta}$
$-\widehat{h}(\eta ; p) \frac{\partial \widehat{f}(\eta ; p)}{\partial \eta}+\beta\left[\widehat{h}(\eta ; p) \frac{\partial^{3} \widehat{f}(\eta ; p)}{\partial \eta^{3}}\right.$
$\left.+\frac{\partial \widehat{f}(\eta ; p)}{\partial \eta} \frac{\partial^{2} \widehat{h}(\eta ; p)}{\partial \eta^{2}}\right]+\beta\left[3 \frac{\partial^{2} \widehat{f}(\eta ; p)}{\partial \eta^{2}} \frac{\partial \widehat{h}(\eta ; p)}{\partial \eta}\right.$
$\left.-\widehat{f}(\eta ; p) \frac{\partial^{3} \widehat{h}(\eta ; p)}{\partial \eta^{3}}\right]+2 A \frac{\partial^{2} \widehat{f}(\eta ; p)}{\partial \eta^{2}} \frac{\partial \widehat{h}(\eta ; p)}{\partial \eta}$
$+6 B K\left(\frac{\partial \widehat{h}(\eta ; p)}{\partial \eta}\right)^{2} \frac{\partial^{2} \widehat{h}(\eta ; p)}{\partial \eta^{2}}$

$\begin{aligned} h_{m}(0) & =0, \quad h_{m}(\infty)=0, \\ \theta_{m}(0) & =0, \quad \theta_{m}(\infty)=0,\end{aligned}$

$\mathcal{R}_{1 m}(\eta)=f_{m-1}^{\prime \prime \prime}(\eta)+\left(1-\chi_{m}\right)$

$+\sum_{k=0}^{m-1}\left[f_{m-1-k} f_{k}^{\prime \prime}-f_{m-1-k}^{\prime} f_{k}^{\prime}\right]+\beta \sum_{k=0}^{m-1}\left[2 f_{m-1-k}^{\prime} f_{k}^{\prime \prime \prime}\right.$

$\left.+3 f_{m-1-k}^{\prime \prime} f_{k}^{\prime \prime}-f_{m-1-k} f_{k}^{\prime \prime \prime \prime}\right]+2 A \sum_{k=0}^{m-1}\left[f_{m-1-k}^{\prime \prime} f_{k}^{\prime \prime}\right]$

$+6 B Z \sum_{k=0}^{m-1}\left[\sum_{p=0}^{m-1} f_{p-1}^{\prime \prime} f_{k-p}^{\prime \prime}\right] h_{m-k}^{\prime \prime}$

$\mathcal{N}_{3}[\widehat{\boldsymbol{\theta}}(\eta ; p), \widehat{f}(\eta ; p), \widehat{h}(\eta ; p)]=\frac{\partial^{2} \widehat{\boldsymbol{\theta}}(\eta ; p)}{\partial \eta^{2}}$

$+6 B S \sum_{k=0}^{m-1}\left[\sum_{p=0}^{m-1} f_{p-1}^{\prime \prime} f_{k-p}^{\prime \prime}\right] f_{m-k}^{\prime \prime \prime}$

$+P_{\mathrm{r}}\left[\widehat{f}(\eta ; p) \frac{\partial \widehat{\theta}(\eta ; p)}{\partial \eta}-2 \widehat{\theta}(\eta ; p) \frac{\partial \widehat{f}(\eta ; p)}{\partial \eta}\right.$

$+6 B K \sum_{k=0}^{m-1}\left[\sum_{p=0}^{m-1} h_{p-1}^{\prime} h_{k-p}^{\prime}\right] f_{m-k}^{\prime \prime \prime}$

$+12 B Z \sum_{k=0}^{m-1}\left[\sum_{p=0}^{m-1} h_{p-1}^{\prime} f_{k-p}^{\prime \prime}\right] f_{m-k}^{\prime \prime \prime}$

$+12 B K \sum_{k=0}^{m-1}\left[\sum_{p=0}^{m-1} h_{p-1}^{\prime} f_{k-p}^{\prime \prime}\right] h_{m-k}^{\prime \prime}$

$\left.+2 M \frac{\partial^{2} \widehat{f}(\eta ; p)}{\partial \eta^{2}} \frac{\partial \widehat{h}(\eta ; p)}{\partial \eta}\right]+\beta P_{\mathrm{r}} E\left[\frac{\partial \widehat{f}(\eta ; p)}{\partial \eta}\right.$

$\left.\cdot\left(\frac{\partial^{2} \widehat{f}(\eta ; p)}{\partial \eta^{2}}\right)^{2}+M \frac{\partial \widehat{f}(\eta ; p)}{\partial \eta} \frac{\partial^{2} \widehat{f}(\eta ; p)}{\partial \eta^{2}} \frac{\partial \widehat{h}(\eta ; p)}{\partial \eta}\right]$

$+\beta P_{\mathrm{r}} E\left[M \widehat{h}(\eta ; p)\left(\frac{\partial^{2} \widehat{f}(\eta ; p)}{\partial \eta^{2}}\right)^{2}-\widehat{f}(\eta ; p) \frac{\partial^{2} \widehat{f}(\eta ; p)}{\partial \eta^{2}}\right.$

$\mathcal{R}_{2 m}(\eta)=h_{m-1}^{\prime \prime}(\eta)+\sum_{k=0}^{m-1}\left[h_{m-1-k}^{\prime} f_{k}-h_{m-1-k} f_{k}^{\prime}\right.$

$\left.+2 A h_{m-1-k}^{\prime} f_{k}^{\prime \prime}\right]+\beta \sum_{k=0}^{m-1}\left[h_{m-1-k} f_{k}^{\prime \prime \prime}+f_{m-1-k}^{\prime} h_{k}^{\prime \prime}\right.$

$\left.+3 h_{m-1-k}^{\prime} f_{k}^{\prime \prime}-f_{m-1-k} h_{k}^{\prime \prime \prime}\right]$

$\left.\cdot \frac{\partial^{3} \widehat{f}(\eta ; p)}{\partial \eta^{3}}\right]-\beta P_{\mathrm{r}} E M\left[\widehat{f}(\eta ; p) \frac{\partial^{2} \widehat{f}(\eta ; p)}{\partial \eta^{2}} \frac{\partial^{2} \widehat{h}(\eta ; p)}{\partial \eta^{2}}\right.$

$\left.+\widehat{f}(\eta ; p) \frac{\partial \widehat{h}(\eta ; p)}{\partial \eta} \frac{\partial^{3} \widehat{f}(\eta ; p)}{\partial \eta^{3}}\right]+2 P_{\mathrm{r}} B E S$

$\cdot\left[\left(\frac{\partial^{2} \widehat{f}(\eta ; p)}{\partial \eta^{2}}\right)^{4}\right]+2 P_{\mathrm{r}} B E S\left[4 M\left(\frac{\partial^{2} \widehat{f}(\eta ; p)}{\partial \eta^{2}}\right)^{3}\right.$

$+6 B K \sum_{k=0}^{m-1}\left[\sum_{p=0}^{m-1} h_{p-1}^{\prime} h_{k-p}^{\prime}\right] h_{m-k}^{\prime \prime}$

$\left.\frac{\partial \widehat{h}(\eta ; p)}{\partial \eta}+6 M^{2}\left(\frac{\partial^{2} \widehat{f}(\eta ; p)}{\partial \eta^{2}}\right)^{2}\left(\frac{\partial \widehat{h}(\eta ; p)}{\partial \eta}\right)^{2}\right]$

$+2 P_{\mathrm{r}} B E S\left[4 M^{3} \frac{\partial^{2} \widehat{f}(\eta ; p)}{\partial \eta^{2}}\left(\frac{\partial \widehat{h}(\eta ; p)}{\partial \eta}\right)^{3}\right]$,

5.1.2. $m$ th-Order Deformation Problems:

$$
\begin{aligned}
& \mathcal{L}_{01}\left[f_{m}(\eta)-\chi_{m} f_{m-1}(\eta)\right]=\hbar_{1} \mathcal{R}_{1 m}(\eta), \\
& \mathcal{L}_{02}\left[h_{m}(\eta)-\chi_{m} h_{m-1}(\eta)\right]=\hbar_{2} \mathcal{R}_{2 m}(\eta), \\
& \mathcal{L}_{03}\left[\theta_{m}(\eta)-\chi_{m} \theta_{m-1}(\eta)\right]=\hbar_{3} \mathcal{R}_{3 m}(\eta), \\
& f_{m}(0)=0, \quad f_{m}^{\prime}(0)=0, \quad f_{m}^{\prime}(\infty)=0,
\end{aligned}
$$

$$
\begin{aligned}
& \mathcal{R}_{3 m}(\eta)=\theta_{m-1}^{\prime \prime}(\eta)+P_{\mathrm{r}} \sum_{k=0}^{m-1}\left[f_{m-1-k} \theta_{k}^{\prime}-2 f_{m-1-k}^{\prime} \theta_{k}\right. \\
& \left.-x f_{m-1-k}^{\prime} \frac{\partial \theta_{k}}{\partial x}-2 M h_{m-1-k} \theta_{k}-M x h_{m-1-k} \frac{\partial \theta_{k}}{\partial x}\right] \\
& +P_{\mathrm{r}} E \sum_{k=0}^{m-1}\left[f_{m-1-k}^{\prime \prime} f_{k}^{\prime \prime}+2 M f_{m-1-k}^{\prime \prime} h_{k}^{\prime}\right] \\
& +\beta P_{\mathrm{r}} E \sum_{k=0}^{m-1}\left[\sum_{p=0}^{m-1} f_{p-1}^{\prime} f_{k-p}^{\prime \prime}\right] f_{m-k}^{\prime \prime} \\
& +\beta P_{\mathrm{r}} E M \sum_{k=0}^{m-1}\left[\sum_{p=0}^{m-1} f_{p-1}^{\prime} f_{k-p}^{\prime \prime}\right] h_{m-k}^{\prime} \\
& +\beta P_{\mathrm{r}} E M \sum_{k=0}^{m-1}\left[\sum_{p=0}^{m-1} h_{p-1} f_{k-p}^{\prime \prime}\right] f_{m-k}^{\prime \prime} \\
& -\beta P_{\mathrm{r}} E \sum_{k=0}^{m-1}\left[\sum_{p=0}^{m-1} f_{p-1} f_{k-p}^{\prime \prime}\right] f_{m-k}^{\prime \prime \prime}
\end{aligned}
$$




$$
\begin{aligned}
& -\beta P_{\mathrm{r}} E M \sum_{k=0}^{m-1}\left[\sum_{p=0}^{m-1} f_{p-1} f_{k-p}^{\prime \prime}\right] h_{m-k}^{\prime \prime} \\
& -\beta P_{\mathrm{r}} E M \sum_{k=0}^{m-1}\left[\sum_{p=0}^{m-1} f_{p-1} h_{k-p}^{\prime}\right] f_{m-k}^{\prime \prime \prime} \\
& +2 P_{\mathrm{r}} B E S\left[f_{m-1-k}^{\prime \prime} \sum_{l=0}^{k} f_{k-l}^{\prime \prime} \sum_{j=0}^{l} f_{l-j}^{\prime \prime} f_{j}^{\prime \prime}\right. \\
& \left.+4 M h_{m-1-k}^{\prime} \sum_{l=0}^{k} f_{k-l}^{\prime \prime} \sum_{j=0}^{l} f_{l-j}^{\prime \prime} f_{j}^{\prime \prime}\right] \\
& +2 P_{\mathrm{r}} B E S\left[6 M^{2} f_{m-1-k}^{\prime \prime} \sum_{l=0}^{k} f_{k-l}^{\prime \prime} \sum_{j=0}^{l} h_{l-j}^{\prime} h_{j}^{\prime}\right. \\
& \left.+4 M^{3} f_{m-1-k}^{\prime \prime} \sum_{l=0}^{k} h_{k-l}^{\prime} \sum_{j=0}^{l} h_{l-j}^{\prime} h_{j}^{\prime}\right], \\
& \chi_{m}= \begin{cases}0, & m \leq 1, \\
1, & m>1 .\end{cases}
\end{aligned}
$$

The symbolic software Mathematica is used to get the solutions of (48) to (50) up to the first few orders of approximations. It is found that the solution for $f$ and $h$ are

$$
\begin{aligned}
f(\eta)= & \sum_{m=0}^{\infty} f_{m}(\eta)=\lim _{M \rightarrow \infty}\left[\sum_{m=0}^{M} a_{m, 0}^{0}\right. \\
& \left.+\sum_{n=1}^{2 M+1} \mathrm{e}^{-(n+1) \eta}\left(\sum_{m=n-1}^{2 M} \sum_{k=1}^{2 m+1-n} a_{m, n}^{k} \eta^{k}\right)\right], \\
h(\eta)= & \sum_{m=0}^{\infty} h_{m}(\eta) \\
=\lim _{M \rightarrow \infty} & {\left[\sum_{n=1}^{2 M+1} \mathrm{e}^{-(n+1) \eta}\left(\sum_{m=n-1}^{2 M} \sum_{k=0}^{2 m+1-n} b_{m, n}^{k} \eta^{k}\right)\right], } \\
\theta(\eta)= & \sum_{m=0}^{\infty} \theta_{m}(\eta) \\
= & \lim _{M \rightarrow \infty}\left[\sum_{n=1}^{2 M+1} \mathrm{e}^{-(n+2) \eta}\left(\sum_{m=n-1}^{2 M} \sum_{k=0}^{2 m+1-n} c_{m, n}^{k} \eta^{k}\right)\right],
\end{aligned}
$$

in which the coefficients $a_{m, n}^{q}, b_{m, n}^{q}$, and $c_{m, n}^{q}$ of $f_{m}(\eta)$, $h_{m}(\eta)$, and $\theta_{m}(\eta)$ can be determined by using the given boundary conditions and by initial guess approximations in (30) to (32). We note that the solution for $\varphi_{m}(\eta)$ is the same as $\theta_{m}(\eta)$ given in (60). The numerical results of the above mentioned solutions are presented by graphs.

\subsection{Axisymmetric Stagnation Flow Towards a Shrinking Surface}

In order to find HAM solutions of (18), (19), and (23), we choose the initial approximations and the linear operators as

$$
\begin{aligned}
& g_{02}(\eta)=(1-\alpha)(\exp (-\eta)-1)+\eta, \\
& n_{02}(\eta)=\exp (-\eta), \\
& \phi_{02}(\eta)=\exp (-\eta), \\
& \mathcal{L}_{04}[\widehat{g}(\eta ; p)]=\frac{\partial^{3} \widehat{g}(\eta ; p)}{\partial \eta^{3}}+\frac{\partial^{2} \widehat{g}(\eta ; p)}{\partial \eta^{2}}, \\
& \mathcal{L}_{05}[\widehat{n}(\eta ; p)]=\frac{\partial^{2} \widehat{n}(\eta ; p)}{\partial \eta^{2}}+\frac{\partial \widehat{n}(\eta ; p)}{\partial \eta}, \\
& \mathcal{L}_{06}[\widehat{\phi}(\eta ; p)]=\frac{\partial^{2} \widehat{\phi}(\eta ; p)}{\partial \eta^{2}}+\frac{\partial \widehat{\phi}(\eta ; p)}{\partial \eta},
\end{aligned}
$$

which satisfy

$$
\begin{aligned}
& \mathcal{L}_{04}\left[C_{8}+C_{9} \eta+C_{10} \mathrm{e}^{-\eta}\right]=0, \\
& \mathcal{L}_{05}\left[C_{11}+C_{12} \mathrm{e}^{-\eta}\right]=0, \\
& \mathcal{L}_{06}\left[C_{13}+C_{14} \mathrm{e}^{-\eta}\right]=0,
\end{aligned}
$$

where $C_{i}(i=8-14)$ are arbitrary constants. Adopting the procedure of the previous subsection we get the

\subsubsection{Zeroth-Order Deformation Problems:}

$$
\begin{aligned}
& (1-p) \mathcal{L}_{04}\left[\widehat{g}(\eta ; p)-g_{02}(\eta)\right]=p \hbar_{4} \mathcal{N}_{4}[\widehat{g}(\eta ; p)], \\
& (1-p) \mathcal{L}_{05}\left[\widehat{n}(\eta ; p)-n_{02}(\eta)\right]= \\
& p \hbar_{5} \mathcal{N}_{5}[\widehat{n}(\eta ; p), \widehat{g}(\eta ; p)] \text {, } \\
& (1-p) \mathcal{L}_{06}\left[\widehat{\phi}(\eta ; p)-\phi_{02}(\eta)\right]= \\
& p \hbar_{6} \mathcal{N}_{6}[\widehat{\phi}(\eta ; p), \widehat{g}(\eta ; p), \widehat{n}(\eta ; p)], \\
& \widehat{g}(0 ; p)=0, \quad \widehat{g}^{\prime}(0 ; p)=\alpha, \quad \widehat{g}^{\prime}(\infty ; p)=1, \\
& \widehat{n}(0 ; p)=1, \quad \widehat{n}(\infty ; p)=0, \\
& \widehat{\phi}(0 ; p)=1, \quad \widehat{\phi}(\infty ; p)=0 \text {, } \\
& \mathcal{N}_{4}[\widehat{g}(\eta ; p)]=\frac{\partial^{3} \widehat{g}(\eta ; p)}{\partial \eta^{3}}+2 \widehat{g}(\eta ; p) \frac{\partial^{2} \widehat{g}(\eta ; p)}{\partial \eta^{2}} \\
& -\left(\frac{\partial \widehat{g}(\eta ; p)}{\partial \eta}\right)^{2}+1+\beta\left[2 \frac{\partial \widehat{g}(\eta ; p)}{\partial \eta} \frac{\partial^{3} \widehat{g}(\eta ; p)}{\partial \eta^{3}}\right. \\
& \left.+3\left(\frac{\partial^{2} \widehat{g}(\eta ; p)}{\partial \eta^{2}}\right)^{2}-2 \widehat{g}(\eta ; p) \frac{\partial^{4} \widehat{g}(\eta ; p)}{\partial \eta^{4}}\right] \\
& +2 A\left(\frac{\partial^{2} \widehat{g}(\eta ; p)}{\partial \eta^{2}}\right)^{2}+6 B S\left(\frac{\partial^{2} \widehat{g}(\eta ; p)}{\partial \eta^{2}}\right)^{2} \frac{\partial^{3} \widehat{g}(\eta ; p)}{\partial \eta^{3}} \\
& +6 B Z\left(\frac{\partial^{2} \widehat{g}(\eta ; p)}{\partial \eta^{2}}\right)^{2} \frac{\partial^{2} \widehat{n}(\eta ; p)}{\partial \eta^{2}}+6 B K \frac{\partial^{3} \widehat{g}(\eta ; p)}{\partial \eta^{3}} \\
& \cdot\left(\frac{\partial \widehat{n}(\eta ; p)}{\partial \eta}\right)^{2}+12 B Z \frac{\partial \widehat{n}(\eta ; p)}{\partial \eta} \frac{\partial^{2} \widehat{g}(\eta ; p)}{\partial \eta^{2}} \frac{\partial^{3} \widehat{g}(\eta ; p)}{\partial \eta^{3}} \\
& +12 B K \frac{\partial \widehat{n}(\eta ; p)}{\partial \eta} \frac{\partial^{2} \widehat{g}(\eta ; p)}{\partial \eta^{2}} \frac{\partial^{2} \widehat{n}(\eta ; p)}{\partial \eta^{2}}
\end{aligned}
$$




$$
\begin{aligned}
& \mathcal{N}_{5}[\widehat{g}(\eta ; p), \widehat{n}(\eta ; p)]=\frac{\partial^{2} \widehat{n}(\eta ; p)}{\partial \eta^{2}}+2 \widehat{g}(\eta ; p) \frac{\partial \widehat{n}(\eta ; p)}{\partial \eta} \quad \mathcal{R}_{4 m}(\eta)=g_{m-1}^{\prime \prime \prime}(\eta)+\left(1-\chi_{m}\right) \\
& -\widehat{n}(\eta ; p) \frac{\partial \widehat{g}(\eta ; p)}{\partial \eta}+\beta\left[\widehat{n}(\eta ; p) \frac{\partial^{3} \widehat{g}(\eta ; p)}{\partial \eta^{3}}\right. \\
& \left.+\frac{\partial \widehat{g}(\eta ; p)}{\partial \eta} \frac{\partial^{2} \widehat{n}(\eta ; p)}{\partial \eta^{2}}\right]+\beta\left[3 \frac{\partial^{2} \widehat{g}(\eta ; p)}{\partial \eta^{2}} \frac{\partial \widehat{n}(\eta ; p)}{\partial \eta}\right. \\
& \left.-2 \widehat{g}(\eta ; p) \frac{\partial^{3} \widehat{n}(\eta ; p)}{\partial \eta^{3}}\right]+2 A \frac{\partial^{2} \widehat{g}(\eta ; p)}{\partial \eta^{2}} \frac{\partial \widehat{n}(\eta ; p)}{\partial \eta} \\
& +6 B K\left(\frac{\partial \widehat{n}(\eta ; p)}{\partial \eta}\right)^{2} \frac{\partial^{2} \widehat{n}(\eta ; p)}{\partial \eta^{2}} \\
& \mathcal{N}_{6}[\widehat{\phi}(\eta ; p), \widehat{g}(\eta ; p), \widehat{n}(\eta ; p)]=\frac{\partial^{2} \widehat{\phi}(\eta ; p)}{\partial \eta^{2}} \\
& -P_{\mathrm{r}}\left[x \frac{\partial \widehat{g}(\eta ; p)}{\partial \eta} \frac{\partial \widehat{\phi}(\eta ; p)}{\partial x}\right]+P_{\mathrm{r}}\left[2 \widehat{g}(\eta ; p) \frac{\partial \widehat{\phi}(\eta ; p)}{\partial \eta}\right. \\
& \left.-2 \widehat{\phi}(\eta ; p) \frac{\partial \widehat{g}(\eta ; p)}{\partial \eta}\right]-P_{\mathrm{r}} M[2 \widehat{n}(\eta ; p) \widehat{\phi}(\eta ; p) \\
& \left.+x \widehat{n}(\eta ; p) \frac{\partial \widehat{\phi}(\eta ; p)}{\partial x}\right]+P_{\mathrm{r}} E\left[\left(\frac{\partial^{2} \widehat{g}(\eta ; p)}{\partial \eta^{2}}\right)^{2}\right. \\
& \left.+2 M \frac{\partial^{2} \widehat{g}(\eta ; p)}{\partial \eta^{2}} \frac{\partial \widehat{n}(\eta ; p)}{\partial \eta}\right]+\beta P_{\mathrm{r}} E\left[\frac{\partial \widehat{g}(\eta ; p)}{\partial \eta}\right. \\
& \left.\cdot\left(\frac{\partial^{2} \widehat{g}(\eta ; p)}{\partial \eta^{2}}\right)^{2}+M \frac{\partial \widehat{g}(\eta ; p)}{\partial \eta} \frac{\partial^{2} \widehat{g}(\eta ; p)}{\partial \eta^{2}} \frac{\partial \widehat{n}(\eta ; p)}{\partial \eta}\right] \\
& +\beta P_{\mathrm{r}} E\left[M \widehat{n}(\eta ; p)\left(\frac{\partial^{2} \widehat{g}(\eta ; p)}{\partial \eta^{2}}\right)^{2}-2 \widehat{g}(\eta ; p) \frac{\partial^{2} \widehat{g}(\eta ; p)}{\partial \eta^{2}}\right. \\
& \left.\cdot \frac{\partial^{3} \widehat{g}(\eta ; p)}{\partial \eta^{3}}\right]-\beta P_{\mathrm{r}} E M\left[2 \widehat{g}(\eta ; p) \frac{\partial^{2} \widehat{g}(\eta ; p)}{\partial \eta^{2}} \frac{\partial^{2} \widehat{n}(\eta ; p)}{\partial \eta^{2}}\right. \\
& \left.+2 \widehat{g}(\eta ; p) \frac{\partial \widehat{n}(\eta ; p)}{\partial \eta} \frac{\partial^{3} \widehat{g}(\eta ; p)}{\partial \eta^{3}}\right]+2 P_{\mathrm{r}} B E S \\
& \cdot\left[\left(\frac{\partial^{2} \widehat{g}(\eta ; p)}{\partial \eta^{2}}\right)^{4}\right]+2 P_{\mathrm{r}} B E S\left[4 M\left(\frac{\partial^{2} \widehat{g}(\eta ; p)}{\partial \eta^{2}}\right)^{3}\right. \\
& \left.\cdot \frac{\partial \widehat{n}(\eta ; p)}{\partial \eta}+6 M^{2}\left(\frac{\partial^{2} \widehat{g}(\eta ; p)}{\partial \eta^{2}}\right)^{2}\left(\frac{\partial \widehat{n}(\eta ; p)}{\partial \eta}\right)^{2}\right] \\
& \cdot+2 P_{\mathrm{r}} B E S\left[4 M^{3} \frac{\partial^{2} \widehat{g}(\eta ; p)}{\partial \eta^{2}}\left(\frac{\partial \widehat{n}(\eta ; p)}{\partial \eta}\right)^{3}\right] \text {, } \\
& \text { 5.2.2. } m \text { th-Order Deformation Problems: } \\
& \mathcal{L}_{04}\left[g_{m}(\eta)-\chi_{m} g_{m-1}(\eta)\right]=\hbar_{4} \mathcal{R}_{4 m}(\eta), \\
& \mathcal{L}_{05}\left[n_{m}(\eta)-\chi_{m} n_{m-1}(\eta)\right]=\hbar_{5} \mathcal{R}_{5 m}(\eta), \\
& \mathcal{L}_{06}\left[\phi_{m}(\eta)-\chi_{m} \phi_{m-1}(\eta)\right]=\hbar_{6} \mathcal{R}_{6 m}(\eta), \\
& g_{m}(0)=0, \quad g_{m}^{\prime}(0)=0, \quad g_{m}^{\prime}(\infty)=0, \\
& n_{m}(0)=0, \quad n_{m}(\infty)=0 \text {, } \\
& \phi_{m}(0)=0, \quad \phi_{m}(\infty)=0 \text {, } \\
& +\sum_{k=0}^{m-1}\left[2 g_{m-1-k} g_{k}^{\prime \prime}-g_{m-1-k}^{\prime} g_{k}^{\prime}\right]+\beta \sum_{k=0}^{m-1}\left[2 g_{m-1-k}^{\prime} g_{k}^{\prime \prime \prime}\right. \\
& \left.+3 g_{m-1-k}^{\prime \prime} g_{k}^{\prime \prime}-2 g_{m-1-k} g_{k}^{\prime \prime \prime \prime}\right]+2 A \sum_{k=0}^{m-1}\left[g_{m-1-k}^{\prime \prime} g_{k}^{\prime \prime}\right] \\
& +6 B Z \sum_{k=0}^{m-1}\left[\sum_{p=0}^{m-1} g_{p-1}^{\prime \prime} g_{k-p}^{\prime \prime}\right] n_{m-k}^{\prime \prime} \\
& +6 B S \sum_{k=0}^{m-1}\left[\sum_{p=0}^{m-1} g_{p-1}^{\prime \prime} g_{k-p}^{\prime \prime}\right] g_{m-k}^{\prime \prime \prime} \\
& +6 B K \sum_{k=0}^{m-1}\left[\sum_{p=0}^{m-1} n_{p-1}^{\prime} n_{k-p}^{\prime}\right] g_{m-k}^{\prime \prime \prime} \\
& +12 B Z \sum_{k=0}^{m-1}\left[\sum_{p=0}^{m-1} n_{p-1}^{\prime} g_{k-p}^{\prime \prime}\right] g_{m-k}^{\prime \prime \prime} \\
& +12 B K \sum_{k=0}^{m-1}\left[\sum_{p=0}^{m-1} n_{p-1}^{\prime} g_{k-p}^{\prime \prime}\right] n_{m-k}^{\prime \prime} \\
& \mathcal{R}_{5 m}(\eta)=n_{m-1}^{\prime \prime}(\eta)+\sum_{k=0}^{m-1}\left[2 n_{m-1-k}^{\prime} g_{k}-n_{m-1-k} g_{k}^{\prime}\right. \\
& \left.+2 A n_{m-1-k}^{\prime} g_{k}^{\prime \prime}\right]+\beta \sum_{k=0}^{m-1}\left[n_{m-1-k} g_{k}^{\prime \prime \prime}+g_{m-1-k}^{\prime} n_{k}^{\prime \prime}\right. \\
& \left.+3 n_{m-1-k}^{\prime} g_{k}^{\prime \prime}-2 g_{m-1-k} n_{k}^{\prime \prime \prime}\right] \\
& +6 B K \sum_{k=0}^{m-1}\left[\sum_{p=0}^{m-1} n_{p-1}^{\prime} n_{k-p}^{\prime}\right] n_{m-k}^{\prime \prime}, \\
& \left.-x g_{m-1-k}^{\prime} \frac{\partial \phi_{k}}{\partial x}-2 M n_{m-1-k} \phi_{k}-M x n_{m-1-k} \frac{\partial \phi_{k}}{\partial x}\right] \\
& +P_{\mathrm{r}} E \sum_{k=0}^{m-1}\left[g_{m-1-k}^{\prime \prime} g_{k}^{\prime \prime}+2 M g_{m-1-k}^{\prime \prime} n_{k}^{\prime}\right] \\
& +\beta P_{\mathrm{r}} E \sum_{k=0}^{m-1}\left[\sum_{p=0}^{m-1} g_{p-1}^{\prime} g_{k-p}^{\prime \prime}\right] g_{m-k}^{\prime \prime} \\
& +\beta P_{\mathrm{r}} E M \sum_{k=0}^{m-1}\left[\sum_{p=0}^{m-1} g_{p-1}^{\prime} g_{k-p}^{\prime \prime}\right] n_{m-k}^{\prime} \\
& +\beta P_{\mathrm{r}} E M \sum_{k=0}^{m-1}\left[\sum_{p=0}^{m-1} n_{p-1} g_{k-p}^{\prime \prime}\right] g_{m-k}^{\prime \prime} \\
& -2 \beta P_{\mathrm{r}} E \sum_{k=0}^{m-1}\left[\sum_{p=0}^{m-1} g_{p-1} g_{k-p}^{\prime \prime}\right] g_{m-k}^{\prime \prime \prime} \\
& -2 \beta P_{\mathrm{r}} E M \sum_{k=0}^{m-1}\left[\sum_{p=0}^{m-1} g_{p-1} g_{k-p}^{\prime \prime}\right] n_{m-k}^{\prime \prime}
\end{aligned}
$$




$$
\begin{aligned}
& -2 \beta P_{\mathrm{r}} E M \sum_{k=0}^{m-1}\left[\sum_{p=0}^{m-1} g_{p-1} n_{k-p}^{\prime}\right] g_{m-k}^{\prime \prime \prime} \\
& +2 P_{\mathrm{r}} B E S\left[g_{m-1-k}^{\prime \prime} \sum_{l=0}^{k} g_{k-l}^{\prime \prime} \sum_{j=0}^{l} g_{l-j}^{\prime \prime} g_{j}^{\prime \prime}\right. \\
& \left.+4 M n_{m-1-k}^{\prime} \sum_{l=0}^{k} g_{k-l}^{\prime \prime} \sum_{j=0}^{l} g_{l-j}^{\prime \prime} g_{j}^{\prime \prime}\right] \\
& +2 P_{\mathrm{r}} B E S\left[6 M^{2} g_{m-1-k}^{\prime \prime} \sum_{l=0}^{k} g_{k-l}^{\prime \prime} \sum_{j=0}^{l} n_{l-j}^{\prime} n_{j}^{\prime}\right. \\
& \left.+4 M^{3} g_{m-1-k}^{\prime \prime} \sum_{l=0}^{k} n_{k-l}^{\prime} \sum_{j=0}^{l} n_{l-j}^{\prime} n_{j}^{\prime}\right],
\end{aligned}
$$

where

$$
\chi_{m}= \begin{cases}0, & m \leq 1 \\ 1, & m>1\end{cases}
$$

and the solutions are

$$
\begin{aligned}
& g(\eta)=\sum_{m=0}^{\infty} g_{m}(\eta)=\lim _{M \rightarrow \infty}\left[\sum_{m=0}^{M} a_{m, 0}^{0}\right. \\
& \left.+\sum_{n=1}^{2 M+1} \mathrm{e}^{-(n+1) \eta}\left(\sum_{m=n-1}^{2 M} \sum_{k=0}^{2 m+1-n} a_{m, n}^{k} \eta^{k}\right)\right], \\
& n(\eta)=\sum_{m=0}^{\infty} n_{m}(\eta) \\
& =\lim _{M \rightarrow \infty}\left[\sum_{n=1}^{2 M+1} \mathrm{e}^{-(n+1) \eta}\left(\sum_{m=n-1}^{2 M} \sum_{k=0}^{2 m+1-n} b_{m, n}^{k} \eta^{k}\right)\right], \\
& \phi(\eta)=\sum_{m=0}^{\infty} \phi_{m}(\eta) \\
& =\lim _{M \rightarrow \infty}\left[\sum_{n=1}^{2 M+1} \mathrm{e}^{-(n+2) \eta}\left(\sum_{m=n-1}^{2 M} \sum_{k=0}^{2 m+1-n} c_{m, n}^{k} \eta^{k}\right)\right],
\end{aligned}
$$

in which the coefficients $a_{m, n}^{q}, b_{m, n}^{q}$, and $c_{m, n}^{q}$ of $g_{m}(\eta)$, $n_{m}(\eta)$, and $\phi(\eta)$ are constants. We observed that the solution for $\psi_{m}(\eta)$ is the same as $\phi_{m}(\eta)$ given in (91).

\section{Results and Discussion}

The analytic solutions of the two problems defined by (5) - (6), (10), (18) - (19), and (23) have been computed. Obviously, the obtained solutions for (29) to (30) and (58) to (60) contain non-zero auxiliary parameters $\hbar_{i}(i=1-8)$ which can adjust and control the convergence of the solutions. The $\hbar$-curves are presented in Figures $1-8$ and they ensure the convergence of the solutions in the admissible range of the values of

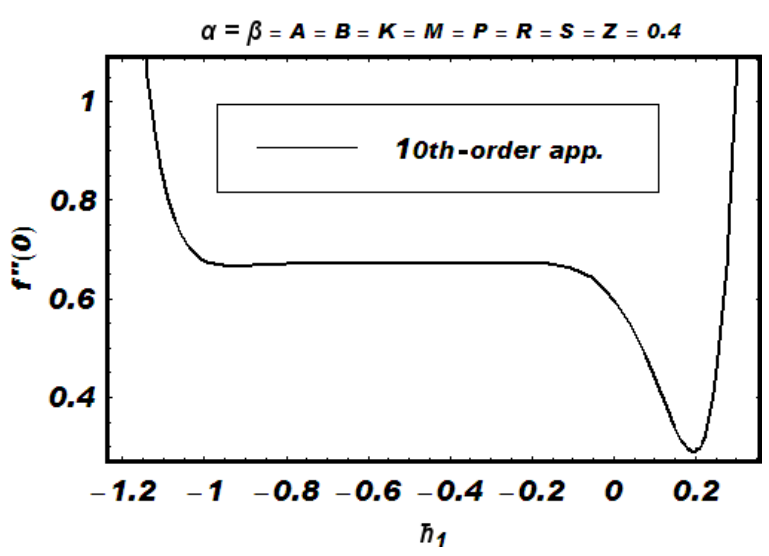

Fig. 1. $\hbar$-curve of $f$ for two-dimensional stagnation flow.

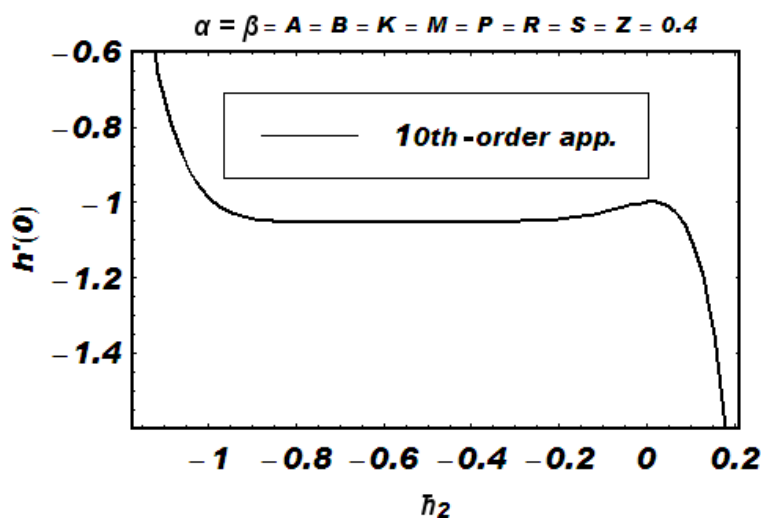

Fig. 2. $\hbar$-curve of $h$ for two-dimensional stagnation flow.

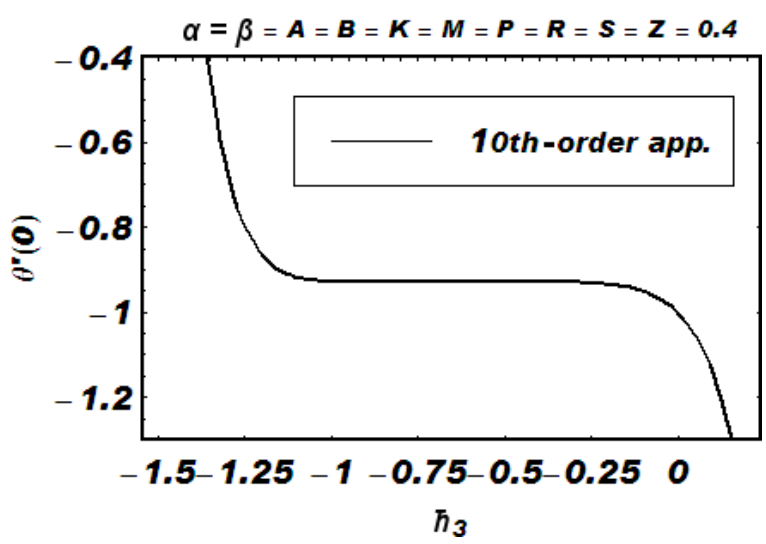

Fig. 3. $\hbar$-curve of $\theta$ for two-dimensional stagnation flow (PST case). 


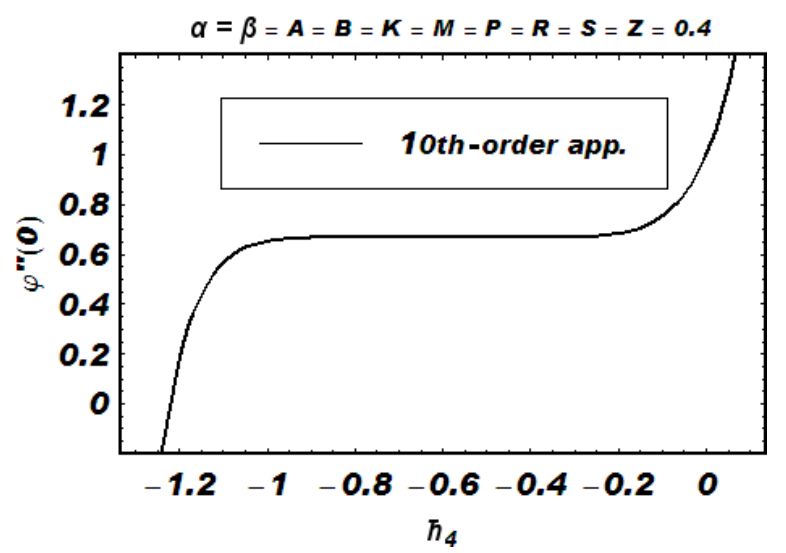

Fig. 4. $\hbar$-curve of $\varphi$ for two-dimensional stagnation flow (PHF case).

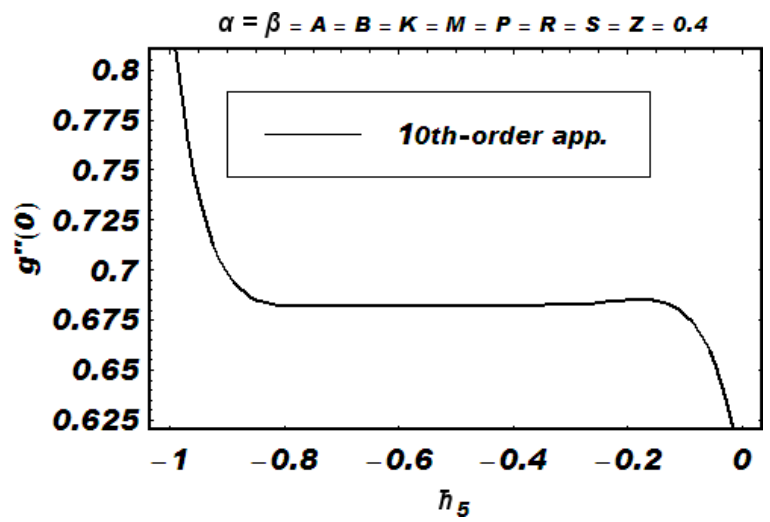

Fig. 5. $\hbar$-curve of $g$ for axisymmetric stagnation flow towards a shrinking surface.

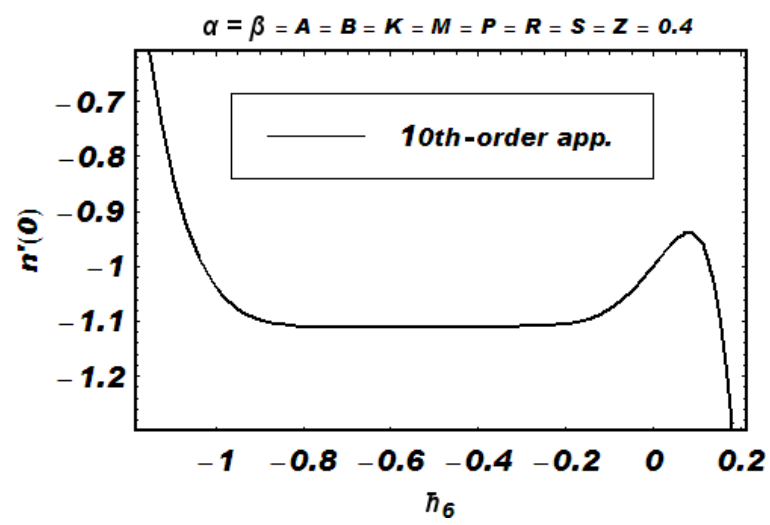

Fig. 6. $\hbar$-curve of $n$ for axisymmetric stagnation flow towards a shrinking surface.

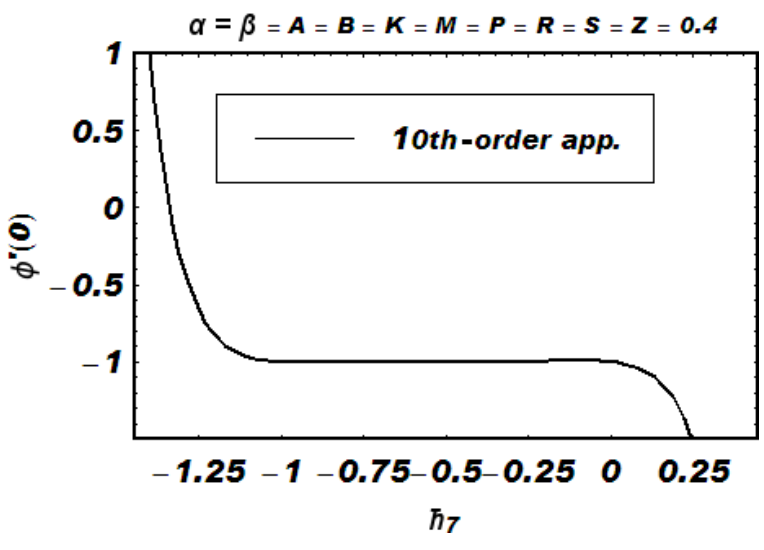

Fig. 7. $\hbar$-curve of $\phi$ for axisymmetric stagnation flow towards a shrinking surface (PST case).

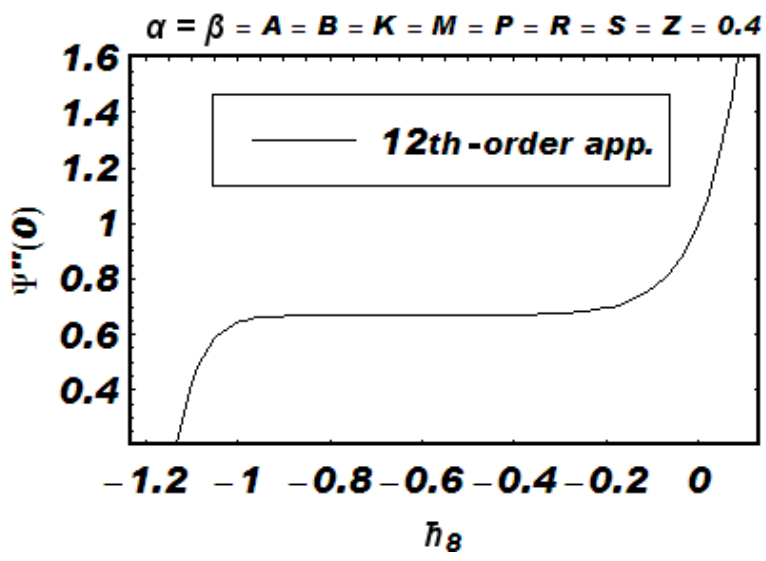

Fig. 8. $\hbar$-curve of $\psi$ for axisymmetric stagnation flow towards a shrinking surface (PHF case).

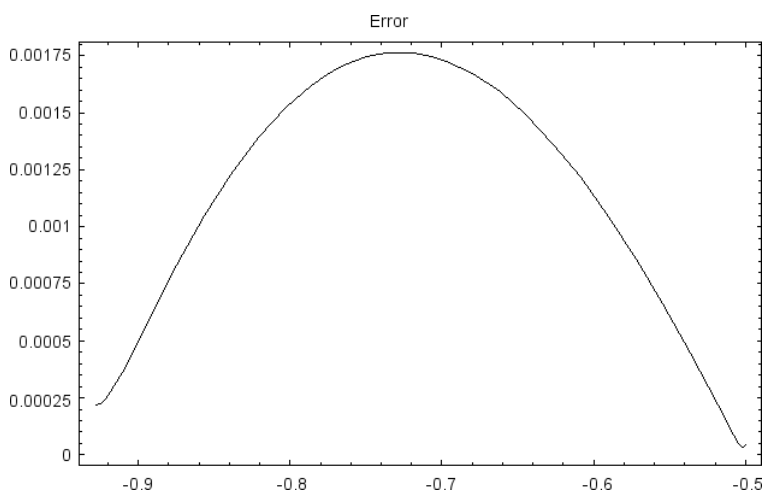

Fig. 9. Residual error of $f$ for two dimensional stagnation flow 


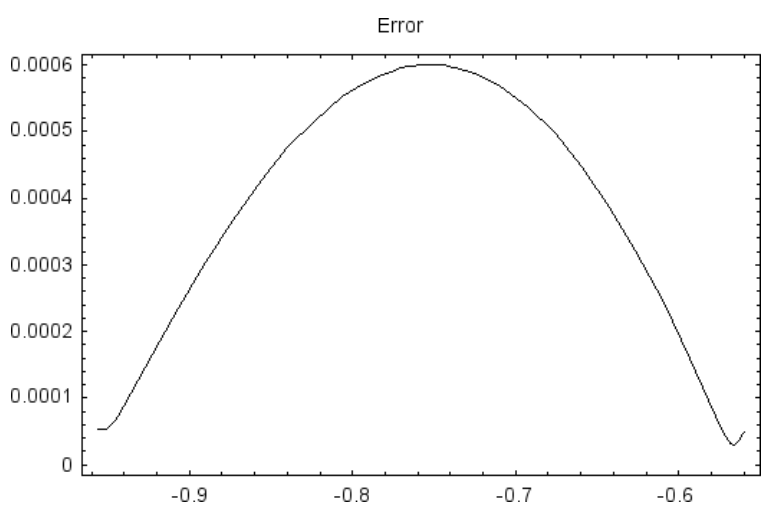

Fig. 10. Residual error of $g$ for axisymmetric stagnation flow towards a shrinking surface.

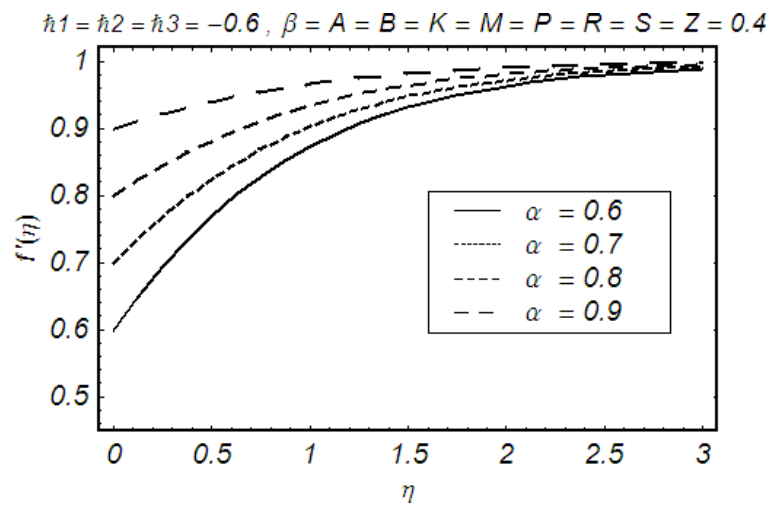

Fig. 11. Influence of stretching parameter $\alpha$ on $f$ for twodimensional stagnation flow.

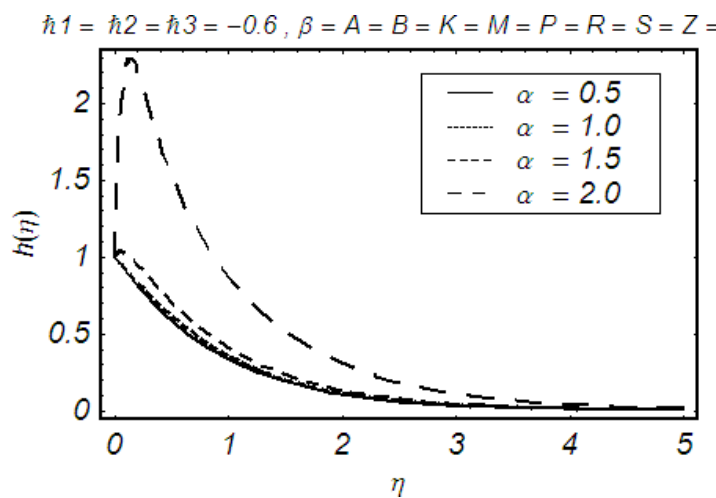

Fig. 12. Influence of stretching parameter $\alpha$ on $h$ for twodimensional stagnation flow.

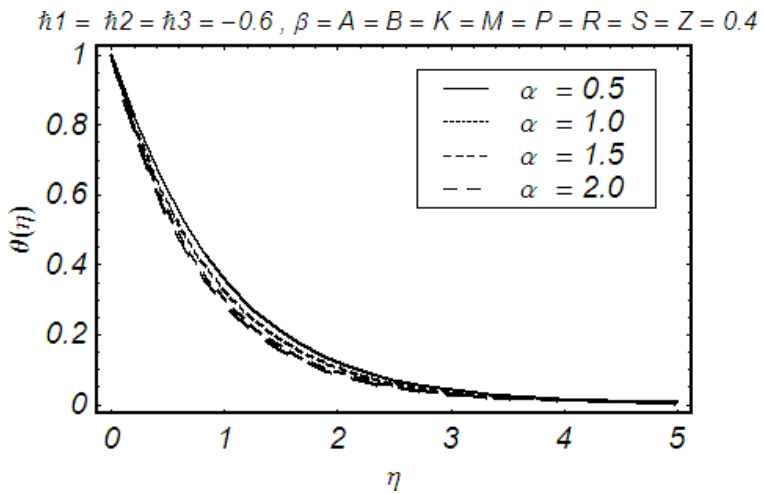

Fig. 13. Influence of stretching parameter $\alpha$ on $\theta$ for twodimensional stagnation flow (PST case).

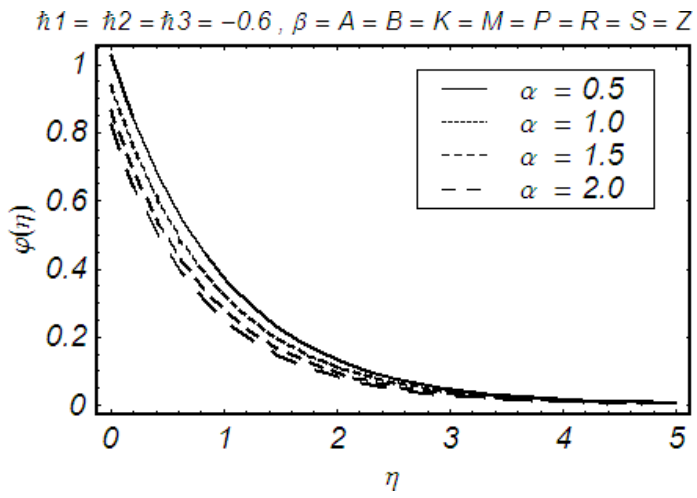

Fig. 14. Influence of stretching parameter $\alpha$ on $\varphi$ for twodimensional stagnation flow (PHF case).

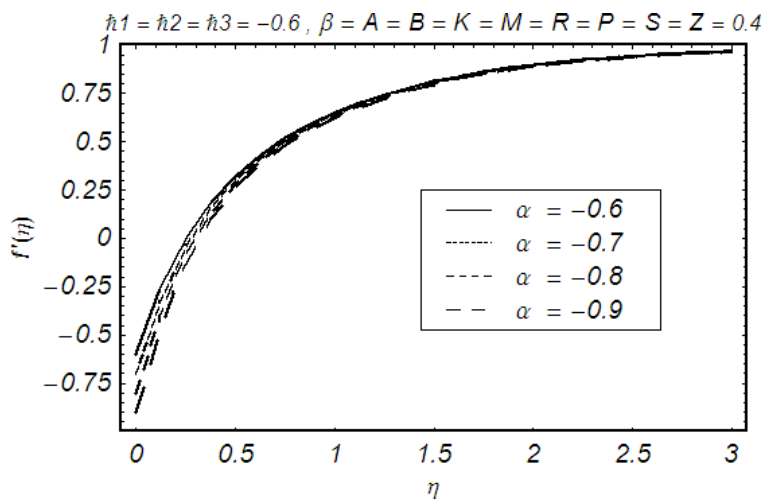

Fig. 15. Influence of shrinking parameter $\alpha$ on $f$ for twodimensional stagnation flow. 


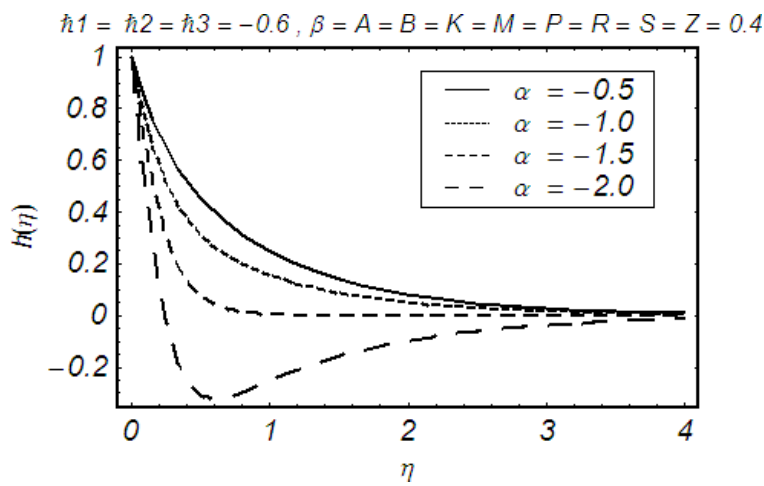

Fig. 16. Influence of shrinking parameter $\alpha$ on $h$ for twodimensional stagnation flow.

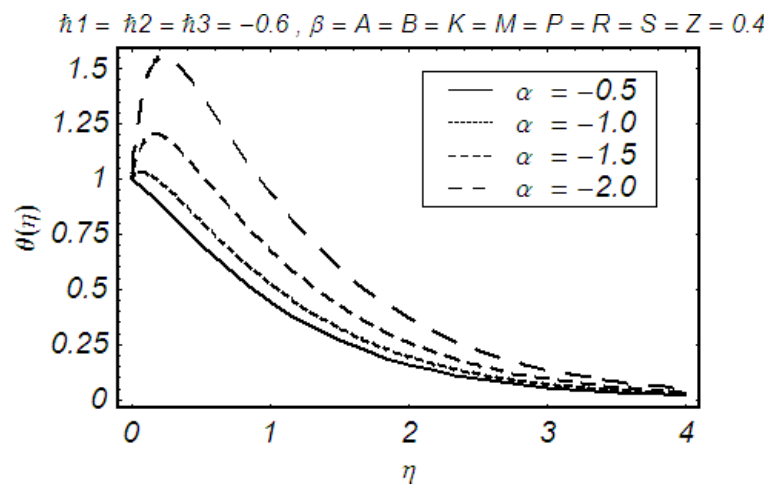

Fig. 17. Influence of shrinking parameter $\alpha$ on $\theta$ for twodimensional stagnation flow (PST case).

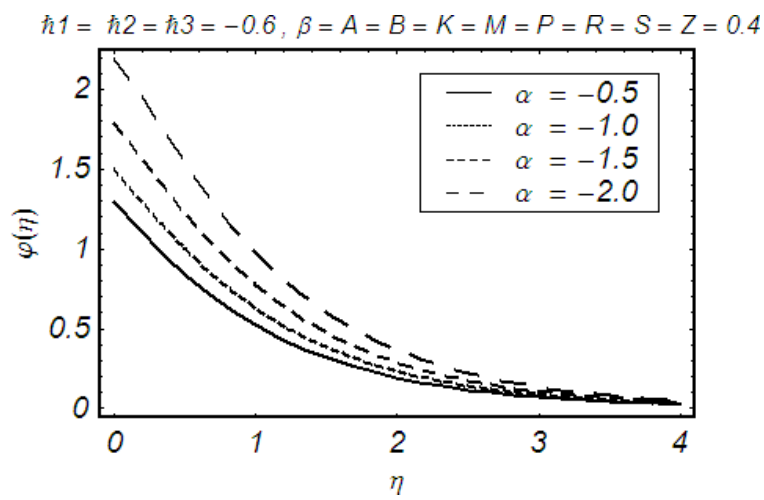

Fig. 18. Influence of shrinking parameter $\alpha$ on $\varphi$ for twodimensional stagnation flow (PHF case) .
Table 1. Homotopy Padé approximation for $f^{\prime \prime}$ when $\alpha=$ $\beta=A=B=S=Z=K=0.1$ for two-dimensional stagnation flow.

\begin{tabular}{ccccccccc}
\hline \multicolumn{8}{c}{ Padé approximands } \\
& {$[1 / 1]$} & {$[2 / 2]$} & {$[3 / 3]$} & {$[4 / 4]$} & {$[5 / 5]$} & {$[6 / 6]$} & {$[7 / 7]$} & {$[8 / 8]$} \\
\hline$f^{\prime \prime}(0)$ & 0.3159 & 0.3255 & 0.3263 & 0.3262 & 0.3262 & 0.3262 & 0.3262 & 0.3262 \\
\hline
\end{tabular}

Table 2. Homotopy Padé approximation for $g^{\prime \prime}$ when $\alpha=$ $\beta=A=B=S=Z=K=0.1$ for axisymmetric stagnation flow towards a shrinking surface.

\begin{tabular}{|c|c|c|c|c|c|c|c|}
\hline & & & adé app & oximan & & & \\
\hline [1/1] & {$[2 / 2]$} & {$[3 / 3]$} & {$[4 / 4]$} & {$[5 / 5]$} & [6/6] & {$[7 / 7]$} & {$[8 / 8]$} \\
\hline 0.319 & 0.321 & 0.32 & .319 & 9311 & 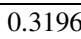 & 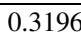 & 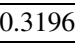 \\
\hline
\end{tabular}

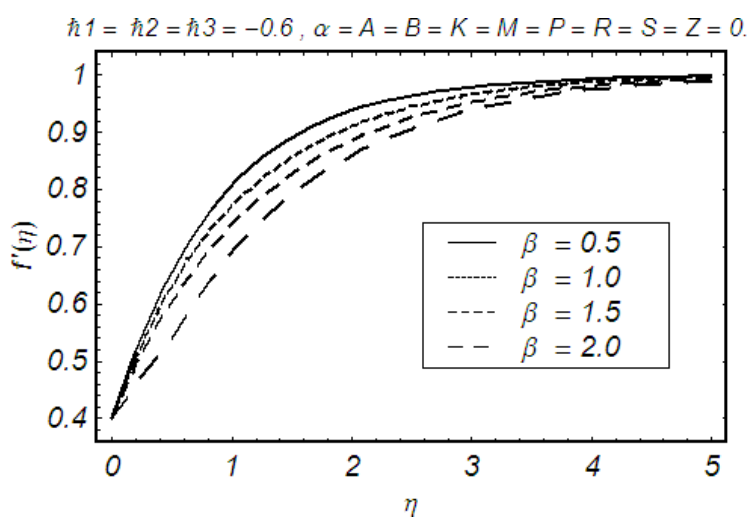

Fig. 19. Influence of non-dimensional parameter $\beta$ on $f$ for two-dimensional stagnation flow.

the auxiliary parameters $\hbar_{i}$. In the present cases, 10th and 12th order of $\hbar_{i}$-curves are plotted in Figures $1-$ 4 for the two-dimensional stagnation flow and in Figures $5-8$ for the axisymmetric stagnation flow. It is seen from these figures that the ranges for the admissible values of $\hbar_{i}$ are $-0.8 \leq \hbar_{1} \leq-0.3,-0.8 \leq \hbar_{2} \leq$ $-0.2,-1 \leq \hbar_{3} \leq-0.2,-0.8 \leq \hbar_{4} \leq-0.3,-0.8 \leq$ $\hbar_{5} \leq-0.4,-0.8 \leq \hbar_{6} \leq-0.3,-1 \leq \hbar_{7} \leq-0.2$, and $-1 \leq \hbar_{8} \leq-0.4$. For the validity of convergence solution the residual errors are also calculated and are shown in Figures 9 and 10. Further, the homotopy Padé approximation solution is also calculated and presented in Tables 1 and 2.

The velocity profiles $f^{\prime}, h$, and the temperature variation that corresponds to $\theta(x, \eta)$ and $\varphi(x, \eta)$ in the PST and PHF case for two-dimensional stagnation flow are plotted in Figures 11-54. The nondimensional velocity $f^{\prime}$ and $h$ against $\eta$ for different values of stretching rate $\alpha>0$ (shrinking rate $\alpha<0$ ) are displayed in Figures 11-14. Here, both $f^{\prime}$ and $h$ increases but $\theta$ and $\phi$ decreases with an increase in the stretching parameter $(\alpha>0)$. The results are quite op- 


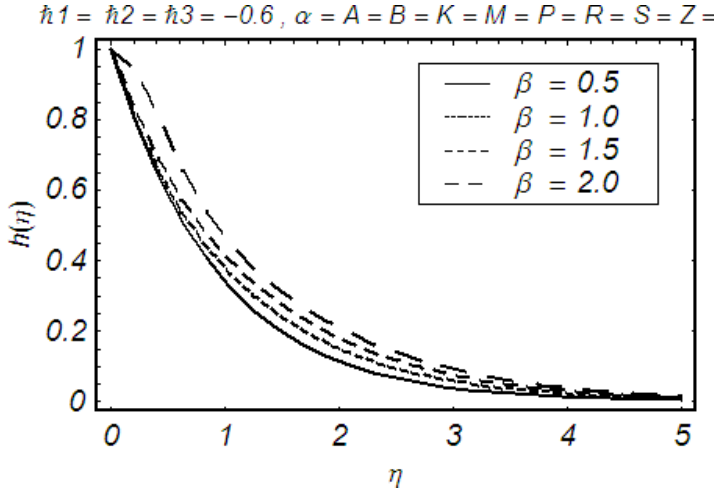

Fig. 20. Influence of non-dimensional parameter $\beta$ on $h$ for two-dimensional stagnation flow.

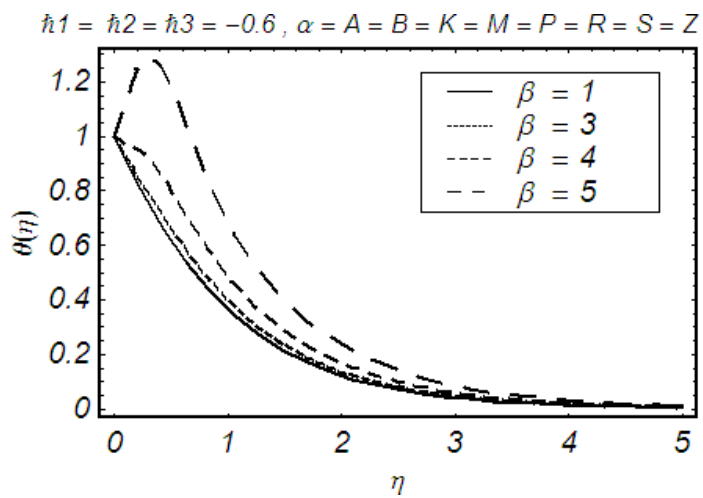

Fig. 21. Influence of non-dimensional parameter $\beta$ on $\theta$ for two-dimensional stagnation flow (PST case).

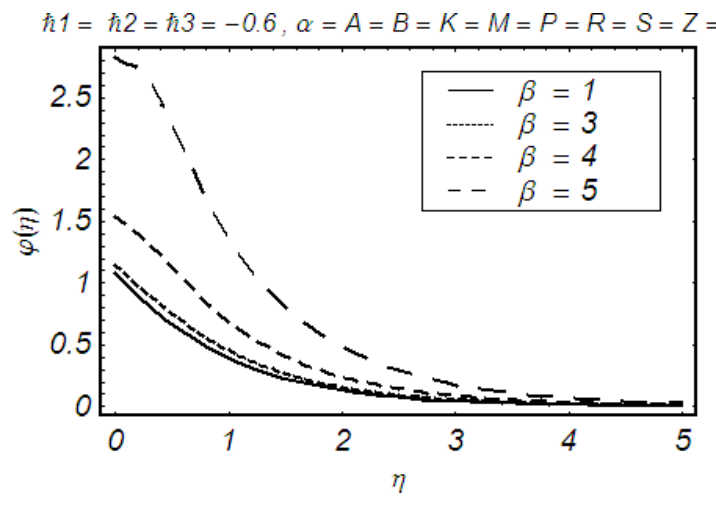

Fig. 22. Influence of non-dimensional parameter $\beta$ on $\varphi$ for two-dimensional stagnation flow (PHF case).

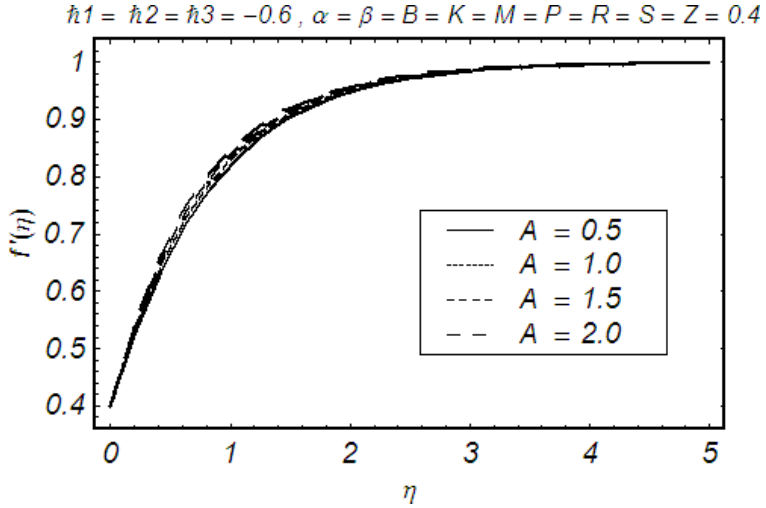

Fig. 23. Influence of non-dimensional parameter $A$ on $f$ for two-dimensional stagnation flow.

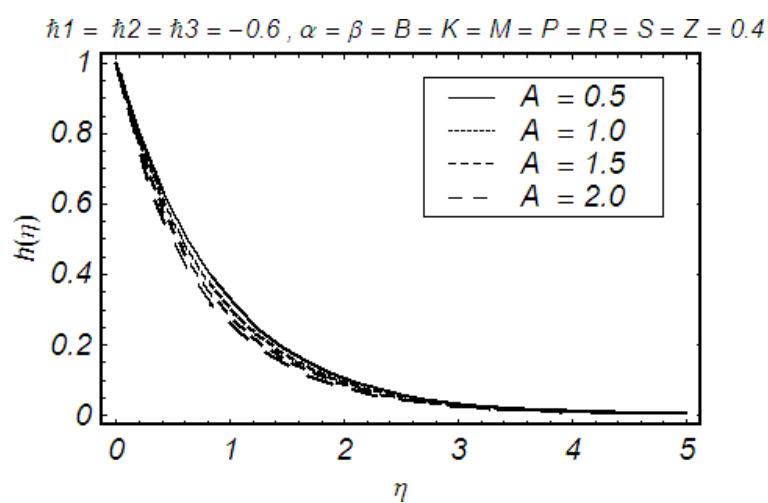

Fig. 24. Influence of non-dimensional parameter $A$ on $h$ for two-dimensional stagnation flow.

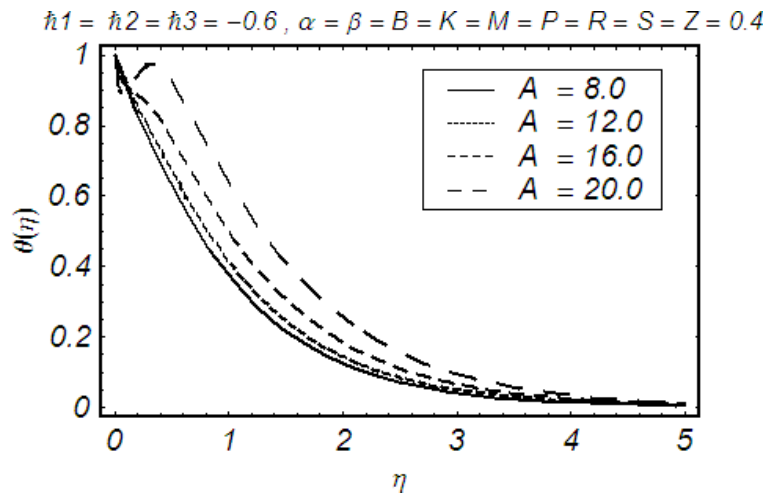

Fig. 25. Influence of non-dimensional parameter $A$ on $\theta$ for two-dimensional stagnation flow (PST case). 


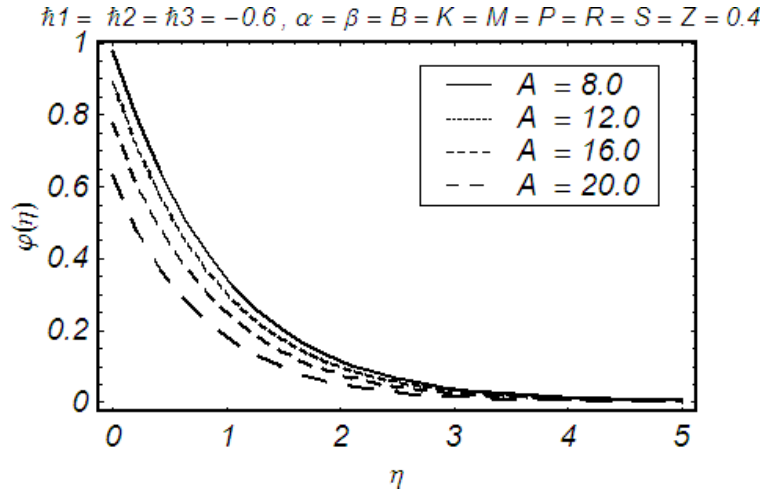

Fig. 26. Influence of non-dimensional parameter $A$ on $\varphi$ for two-dimensional stagnation flow (PHF case).

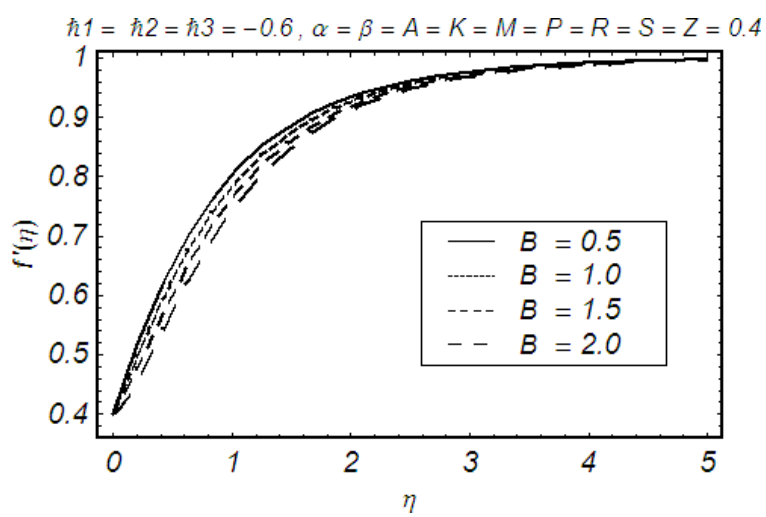

Fig. 27. Influence of non-dimensional parameter $B$ on $f$ for two-dimensional stagnation flow.

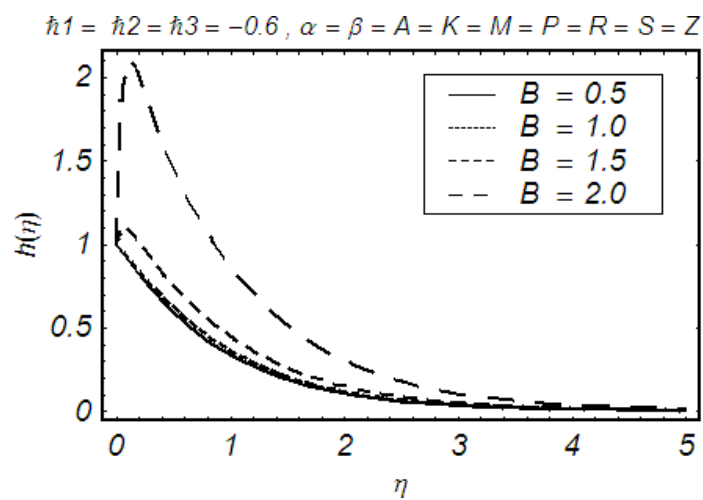

Fig. 28. Influence of non-dimensional parameter $B$ on $h$ for two-dimensional stagnation flow.

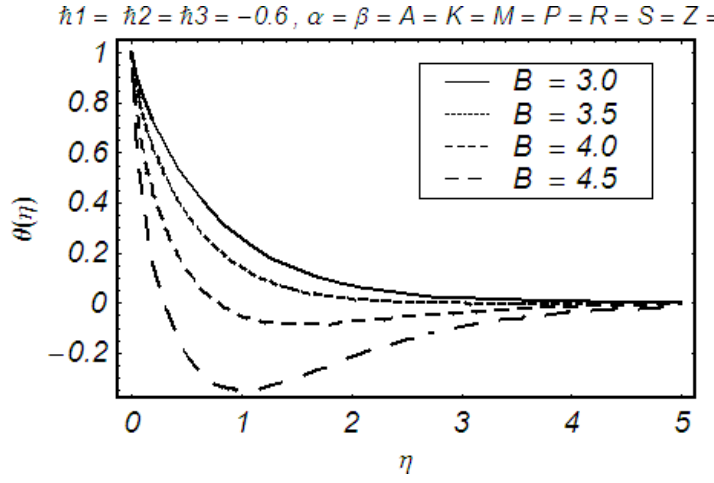

Fig. 29. Influence of non-dimensional parameter $B$ on $\theta$ for two-dimensional stagnation flow (PST case).

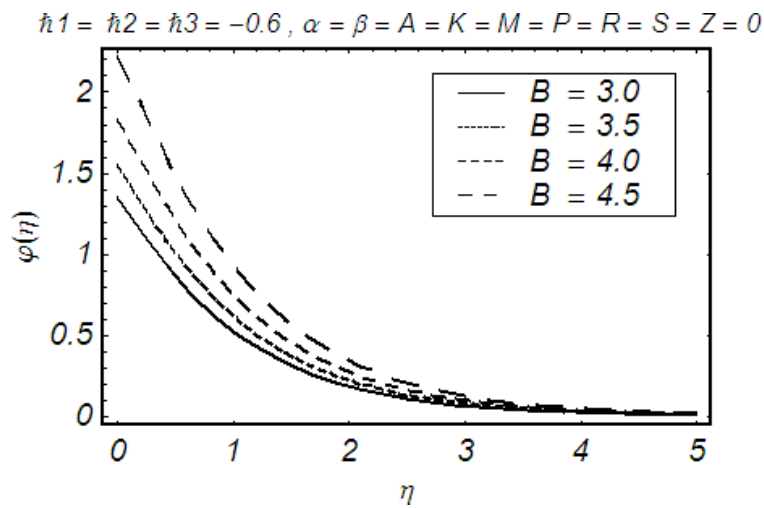

Fig. 30. Influence of non-dimensional parameter $B$ on $\varphi$ for two-dimensional stagnation flow (PHF case).

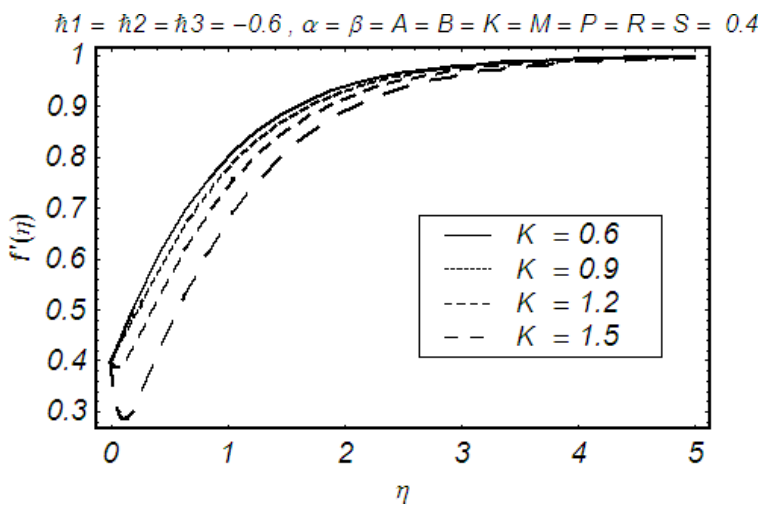

Fig. 31. Influence of non-dimensional parameter $K$ on $f$ for two-dimensional stagnation flow. 


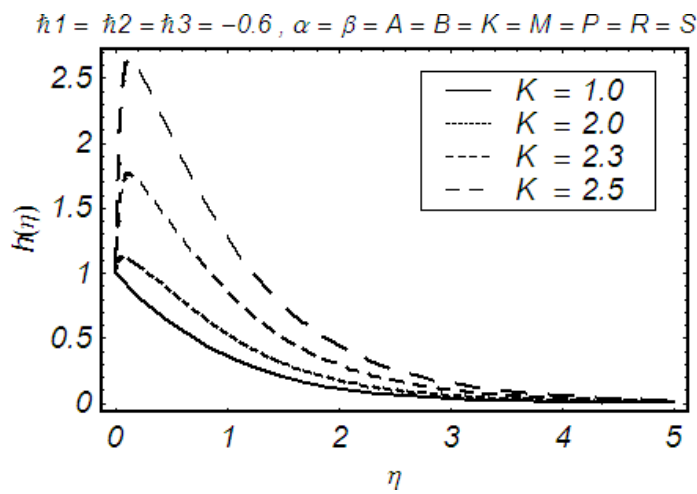

Fig. 32. Influence of non-dimensional parameter $K$ on $h$ for two-dimensional stagnation flow.

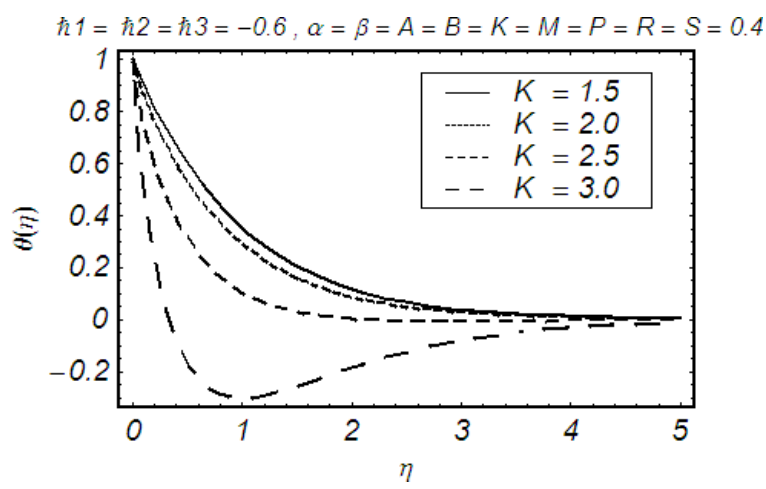

Fig. 33. Influence of non-dimensional parameter $K$ on $\theta$ for two-dimensional stagnation flow (PST case).

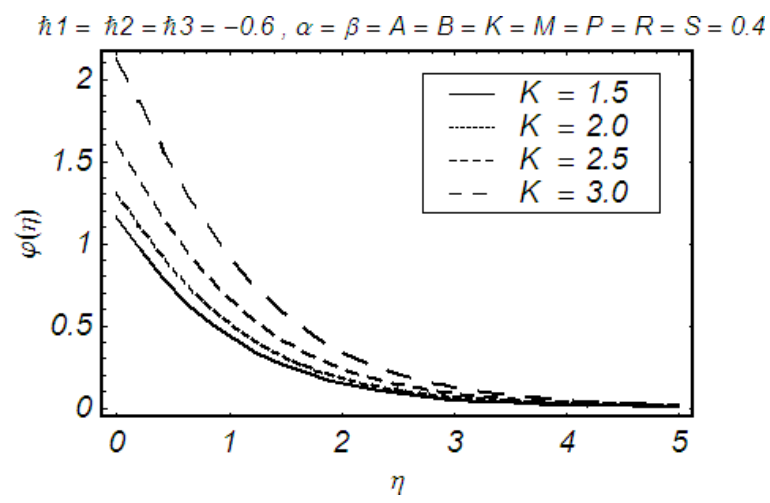

Fig. 34. Influence of non-dimensional parameter $K$ on $\varphi$ for two-dimensional stagnation flow (PHF case).

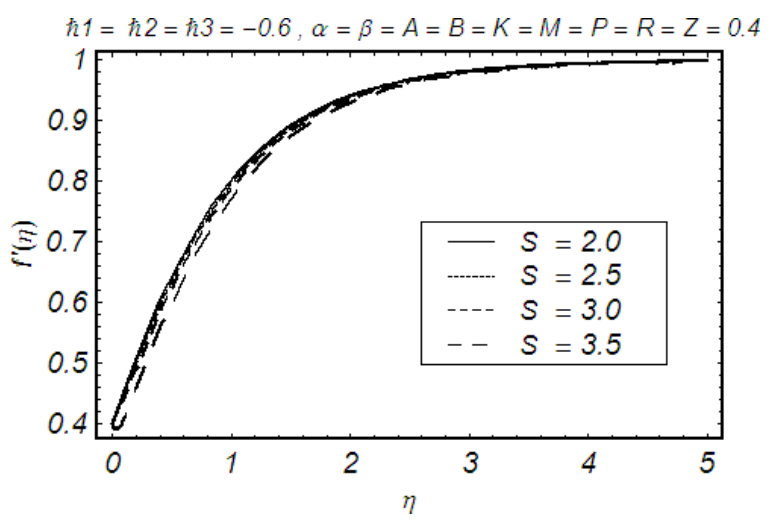

Fig. 35. Influence of non-dimensional parameter $S$ on $f$ for two-dimensional stagnation flow.

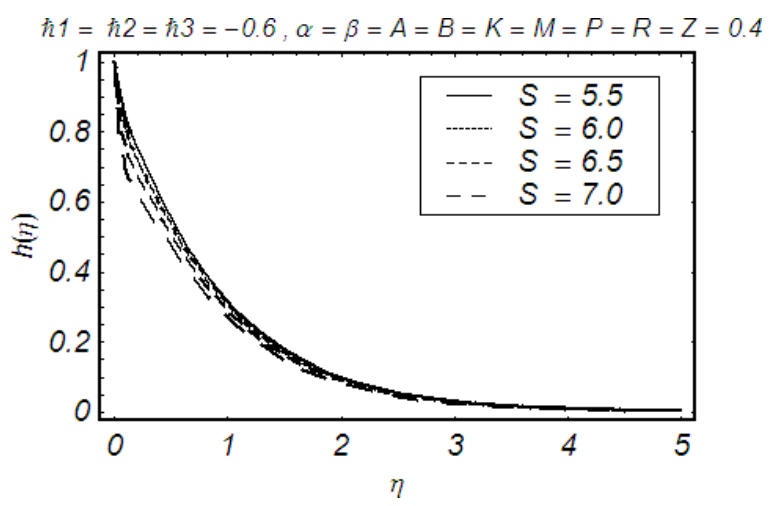

Fig. 36. Influence of non-dimensional parameter $S$ on $h$ for two-dimensional stagnation flow.

posite for the case for shrinking $(\alpha<0)$, which are shown in Figures 15-18. The effect of the nondimensional parameter $\beta$ on $f^{\prime}, h, \theta$, and $\varphi$ are depicted in the Figures $19-22$. It is noticed that for large $\beta, f^{\prime}$ decreases while $h, \theta$, and $\varphi$ increases. The behaviour of the non-dimensional parameter $A$ on $f^{\prime}, h$, $\theta$, and $\varphi$ are shown in Figures 23-26, which gives increase in $f^{\prime}, \varphi$ and decrease in $h, \varphi$ with an increase in $A$. The results are quite opposite for the non-dimensional parameters $B$ and $K$ which are shown in Figures 27-34. There is a decrease in $f^{\prime}, h, \theta$ and increase in $\varphi$ with an increase in $S$ (see Figs. 35 and 38). Figure 39 show increase in $f^{\prime}$ and Figures $40-$ 42 show decrease in $h, \theta, \varphi$ with an increase in the non-dimensional parameter $Z$. Figures $43-44$ show no change in $f^{\prime}, h$ and Figures $45-46$ indicate a decrease in $\theta$ and $\varphi$ with an increase in the Eckert number $E$. The results are similar for non-dimensional parameter $M$ which are shown in Figures 47-50. No change 


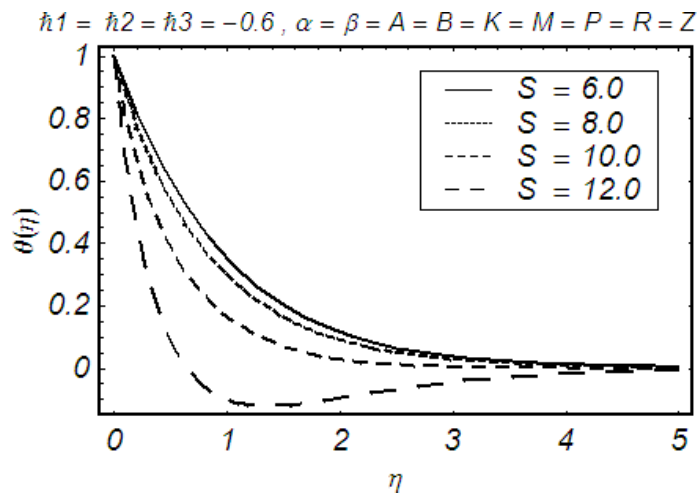

Fig. 37. Influence of non-dimensional parameter $S$ on $\theta$ for two-dimensional stagnation flow (PST case).

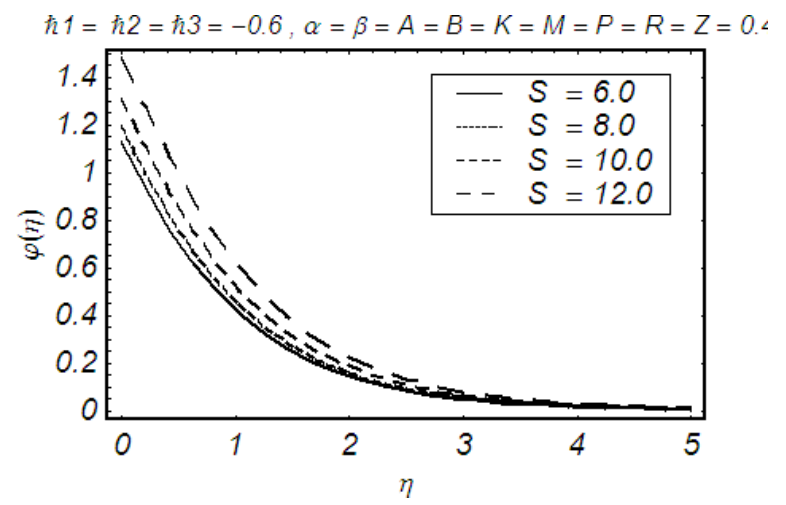

Fig. 38. Influence of non-dimensional parameter $S$ on $\varphi$ for two-dimensional stagnation flow (PHF case).

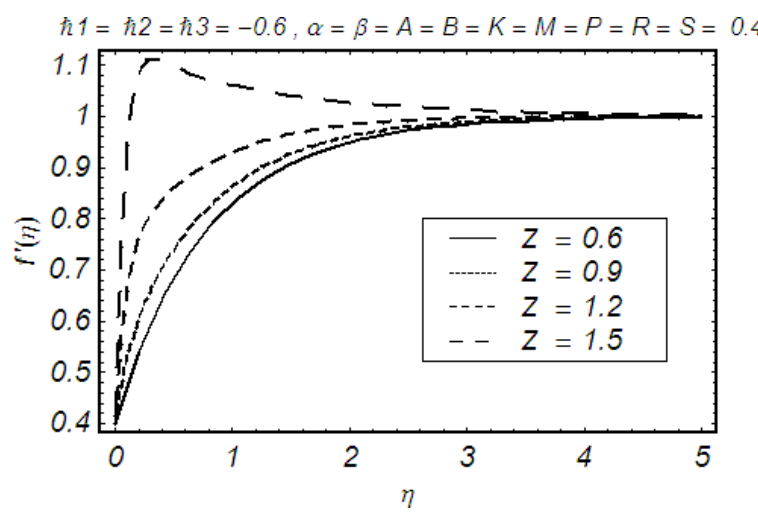

Fig. 39. Influence of non-dimensional parameter $Z$ on $f$ for two-dimensional stagnation flow.

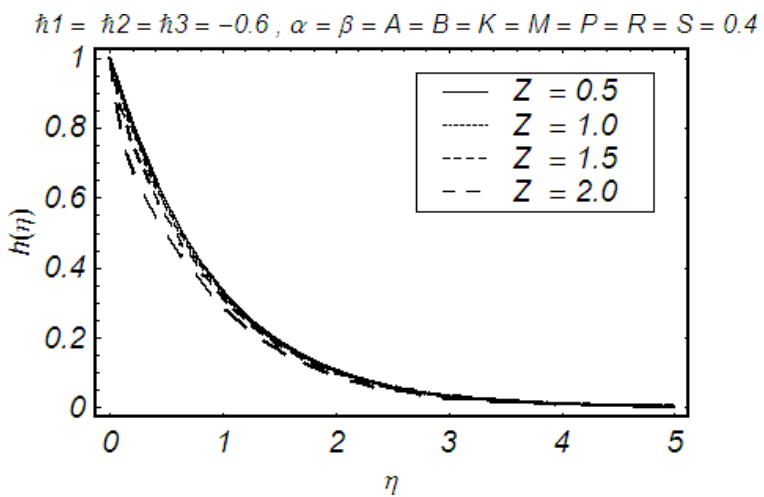

Fig. 40. Influence of non-dimensional parameter $Z$ on $h$ for two-dimensional stagnation flow.

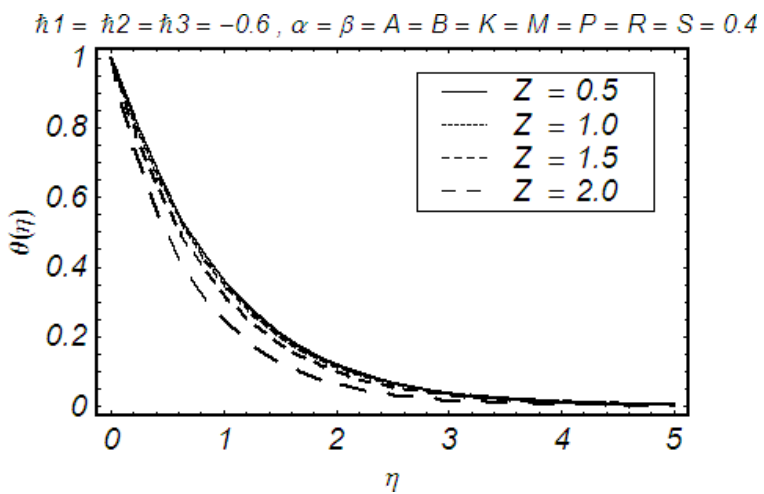

Fig. 41. Influence of non-dimensional parameter $Z$ on $\theta$ for two-dimensional stagnation flow (PST case).

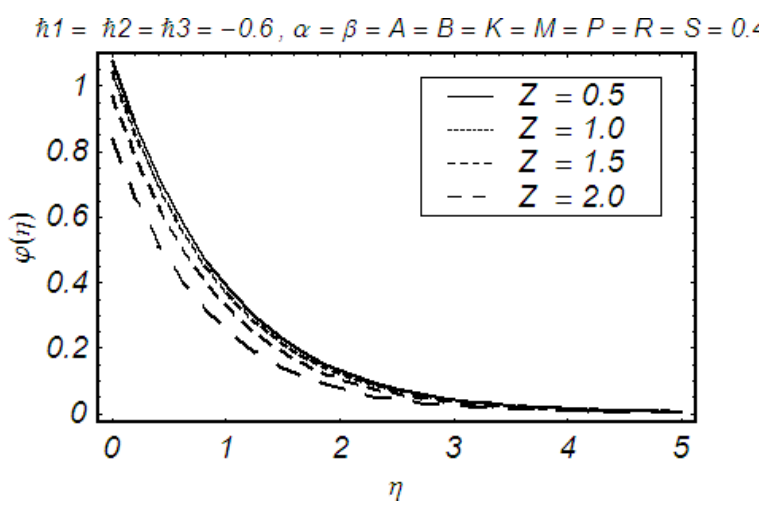

Fig. 42. Influence of non-dimensional parameter $Z$ on $\varphi$ for two-dimensional stagnation flow (PHF case). 


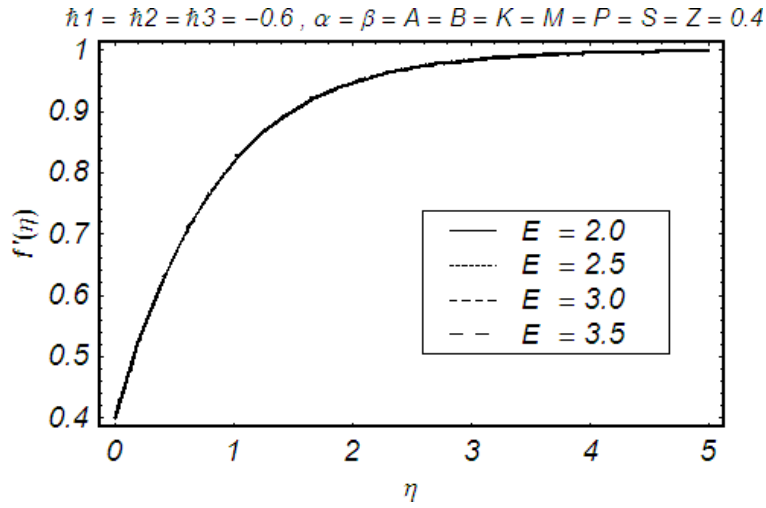

Fig. 43. Influence of Eckert number $E$ on $f$ for twodimensional stagnation flow.

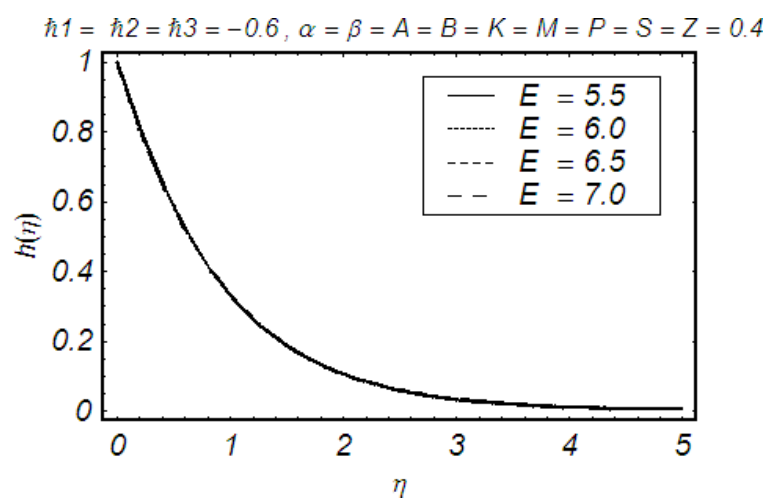

Fig. 44. Influence of Eckert number $E$ on $h$ for twodimensional stagnation flow.

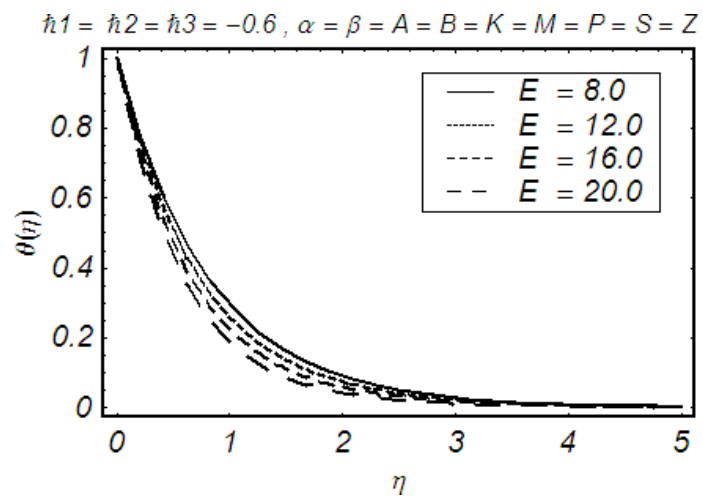

Fig. 45. Influence of Eckert number $E$ on $\theta$ for twodimensional stagnation flow (PST case).

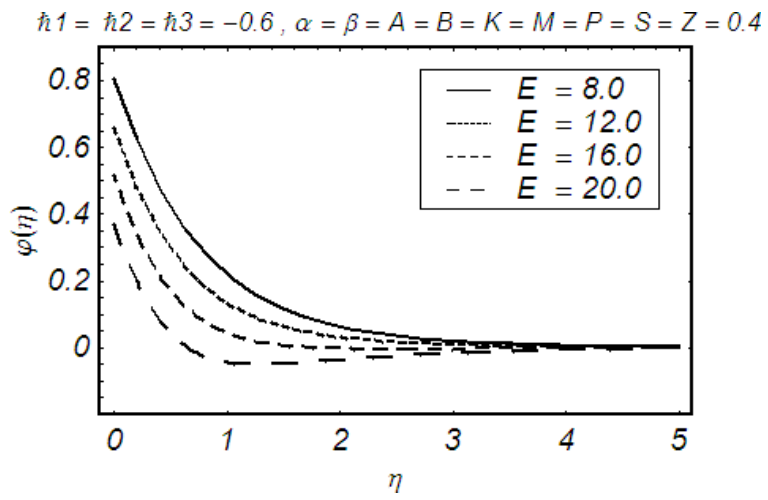

Fig. 46. Influence of Eckert number $E$ on $\varphi$ for twodimensional stagnation flow (PHF case).

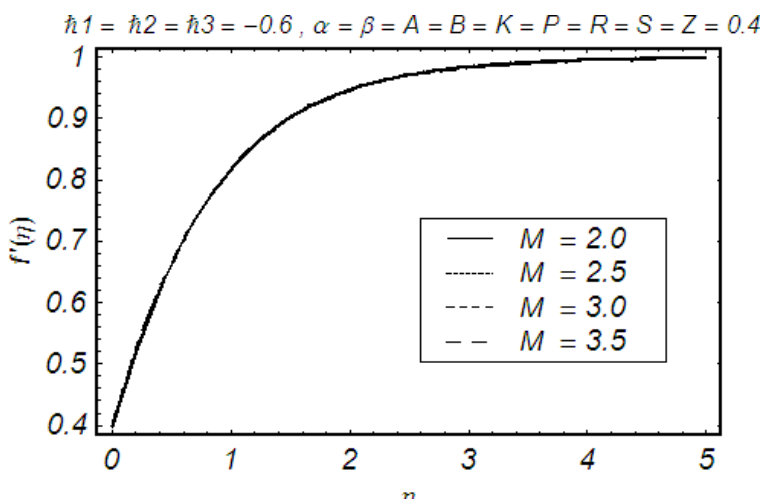

Fig. 47. Influence of non-dimensional parameter $M$ on $f$ for two-dimensional stagnation flow.

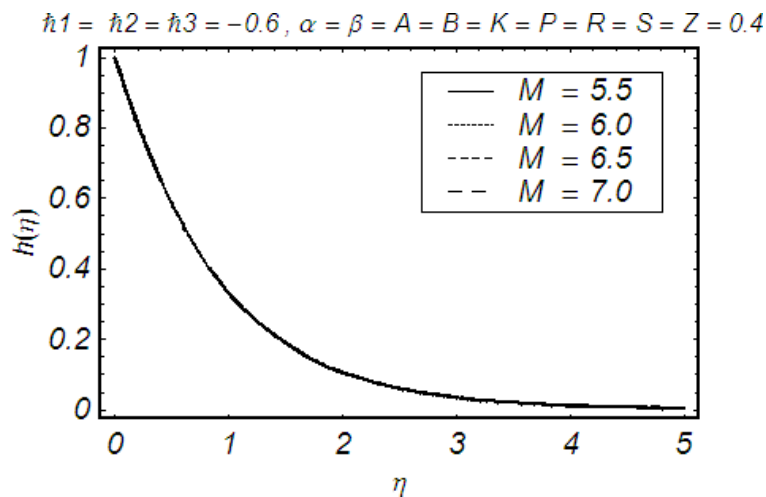

Fig. 48. Influence of non-dimensional parameter $M$ on $h$ for two-dimensional stagnation flow. 


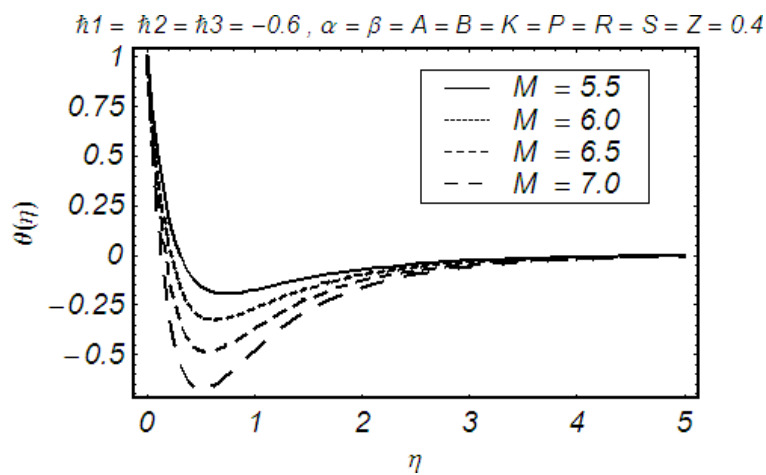

Fig. 49. Influence of non-dimensional parameter $M$ on $\theta$ for two-dimensional stagnation flow (PST case).

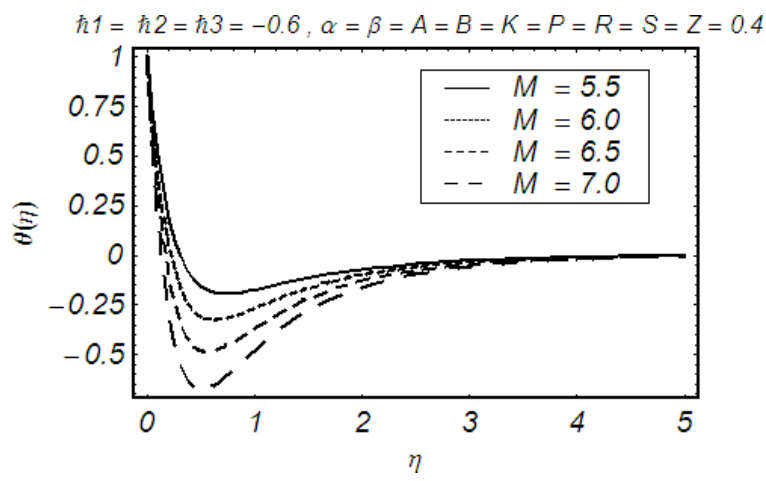

Fig. 50. Influence of non-dimensional parameter $M$ on $\varphi$ for two-dimensional stagnation flow (PHF case).

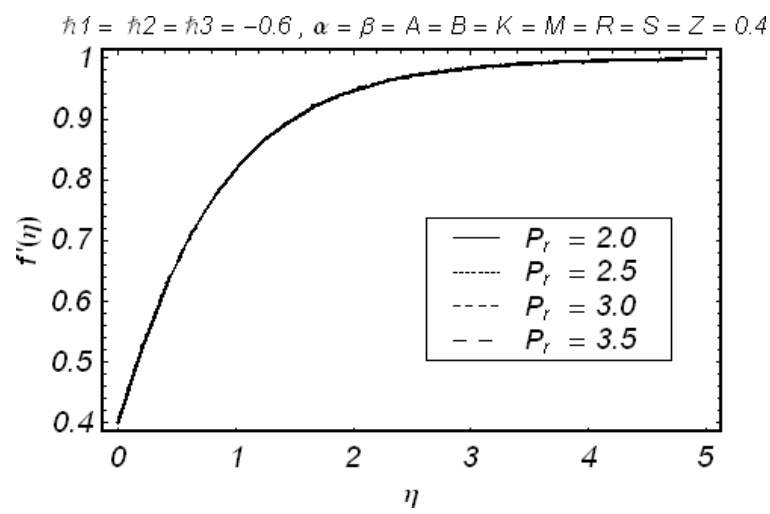

Fig. 51. Influence of Prandtl number $P_{\mathrm{r}}$ on $f$ for twodimensional stagnation flow.

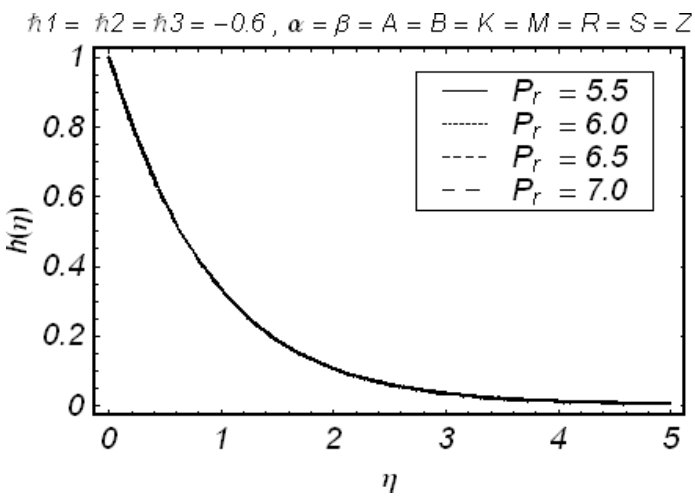

Fig. 52. Influence of Prandtl number $P_{\mathrm{r}}$ on $h$ for twodimensional stagnation flow.

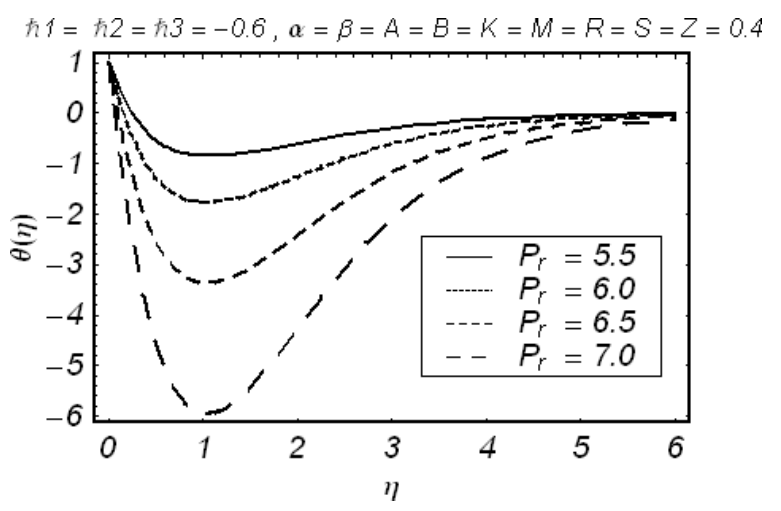

Fig. 53. Influence of Prandtl number $P_{\mathrm{r}}$ on $\theta$ for twodimensional stagnation flow (PST case).

has been found in $f^{\prime}, h$; while $\theta$ and $\varphi$ show opposite behaviour with the increase in Prandtl number $P_{\mathrm{r}}$ (see Figs. 51-54). Figures 55-98 depict $g^{\prime}, n, \phi$, and $\psi$ against $\eta$ for various values of the non-dimensional parameters $\alpha, \beta, A, B, K, S, E, M, P_{\mathrm{r}}$, and $Z$ for the axisymmetric stagnation flow. It is shown that both $g^{\prime}$ and $n$ increases (see Figs. 55-56) but $\phi$ and $\psi$ decreases with an increase in the stretching parameter $(\alpha>0)$ (see Figs. 57-58). The results are quite opposite for the case of shrinking $(\alpha<0)$. These are shown in Figures 59-62. The behaviour of the non-dimensional parameter $A$ on $g^{\prime}, n, \phi$, and $\psi$ are shown in Figures $63-$ 66, which give an increase in $g^{\prime}, \phi$ and a decrease in $n$, $\psi$ with an increase in $A$. The results are quite opposite for the non-dimensional parameters $B$ and $K$, which are shown in Figures $67-70$ and $79-82$. There is a decrease in $g^{\prime}, n, \phi$ and an increase in $\psi$ with an increase in $S$ (see Figs. 91 and 94). Figure 95 reflects an increase in $g^{\prime}$ and Figures 96-98 show a decrease in $n, \phi, \psi$ with an increase in the non-dimensional 


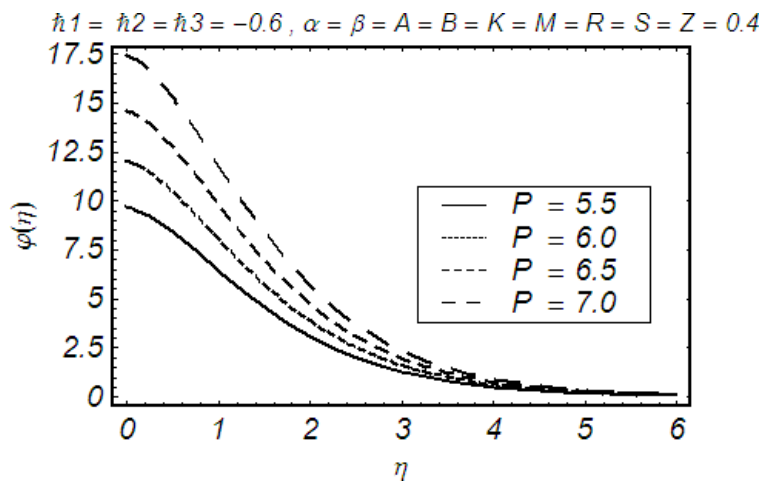

Fig. 54. Influence of Prandtl number $P_{\mathrm{r}}$ on $\varphi$ for twodimensional stagnation flow (PHF case).

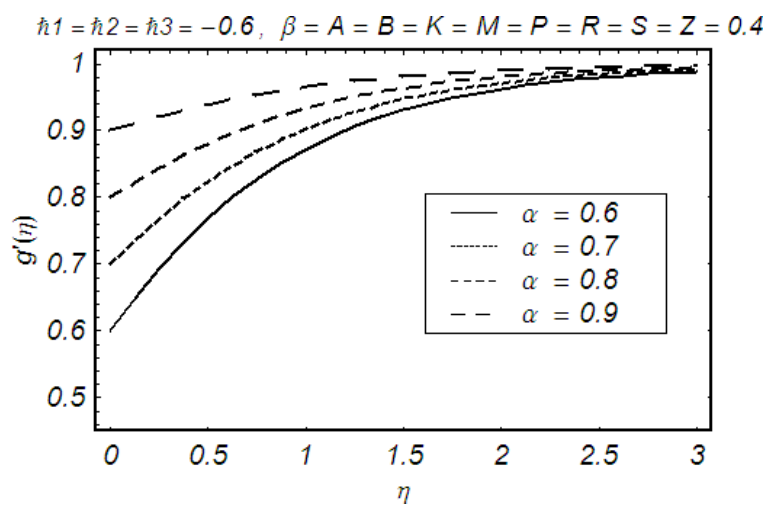

Fig. 55. Influence of stretching parameter $\alpha$ on $g$ for axisymmetric stagnation flow towards a shrinking surface.

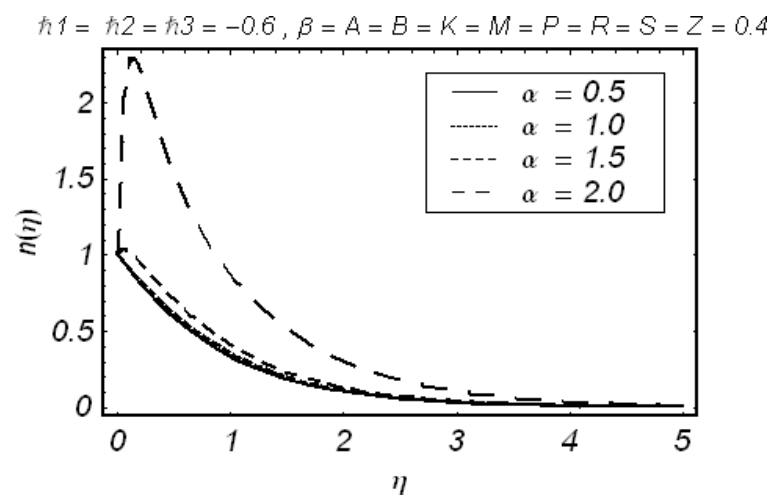

Fig. 56. Influence of stretching parameter $\alpha$ on $n$ for axisymmetric stagnation flow towards a shrinking surface.

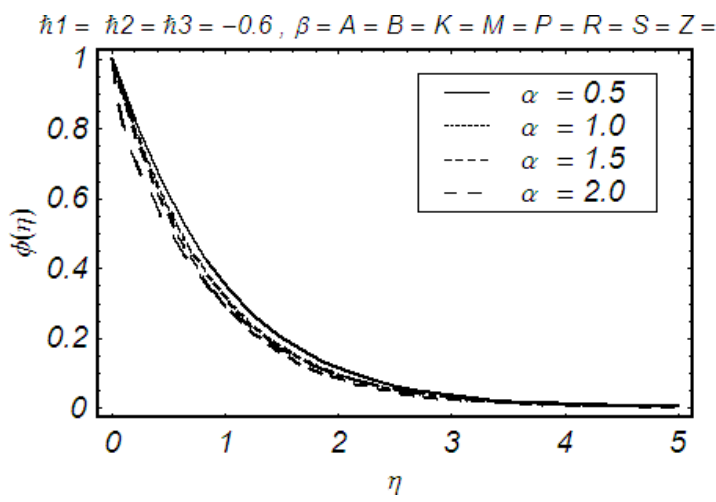

Fig. 57. Influence of stretching parameter $\alpha$ on $\phi$ for axisymmetric stagnation flow towards a shrinking surface (PST case).

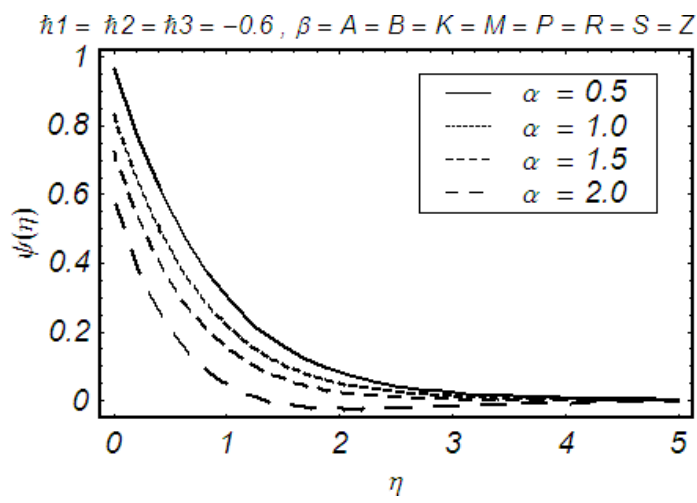

Fig. 58. Influence of stretching parameter $\alpha$ on $\psi$ for axisymmetric stagnation flow towards a shrinking surface (PHF case).

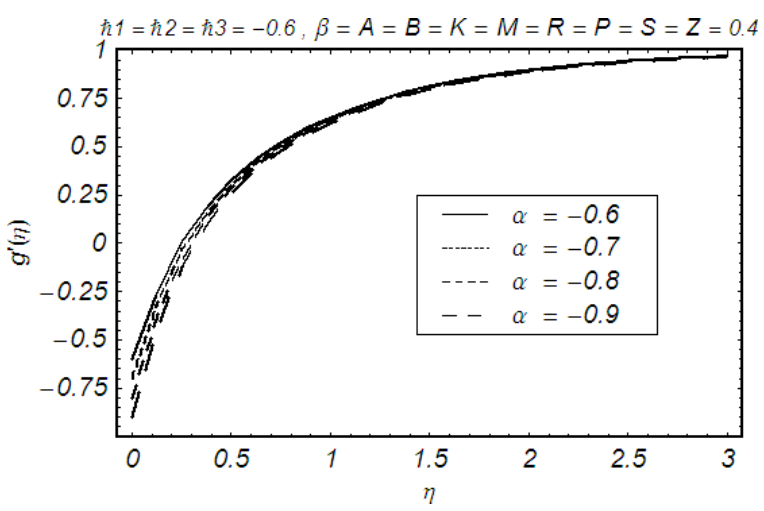

Fig. 59. Influence of shrinking parameter $\alpha$ on $g$ for axisymmetric stagnation flow towards a shrinking surface. 


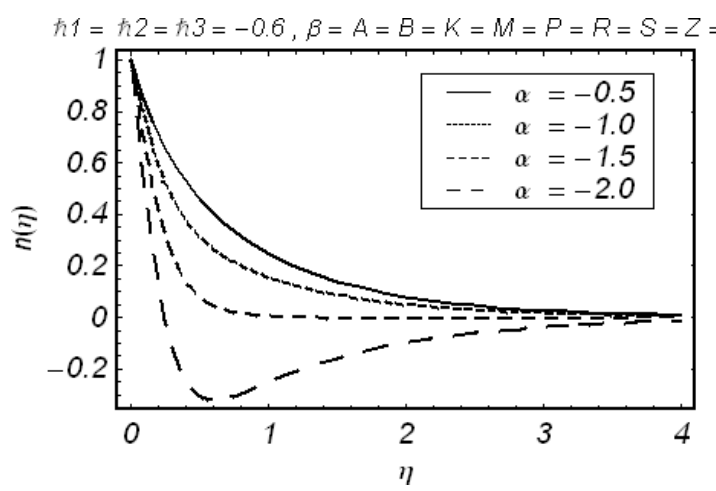

Fig. 60. Influence of shrinking parameter $\alpha$ on $n$ for axisymmetric stagnation flow towards a shrinking surface.

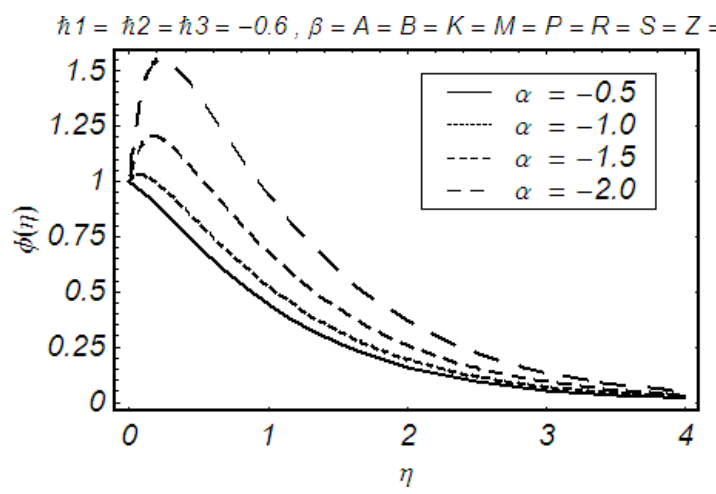

Fig. 61. Influence of shrinking parameter $\alpha$ on $\phi$ for axisymmetric stagnation flow towards a shrinking surface (PST case).

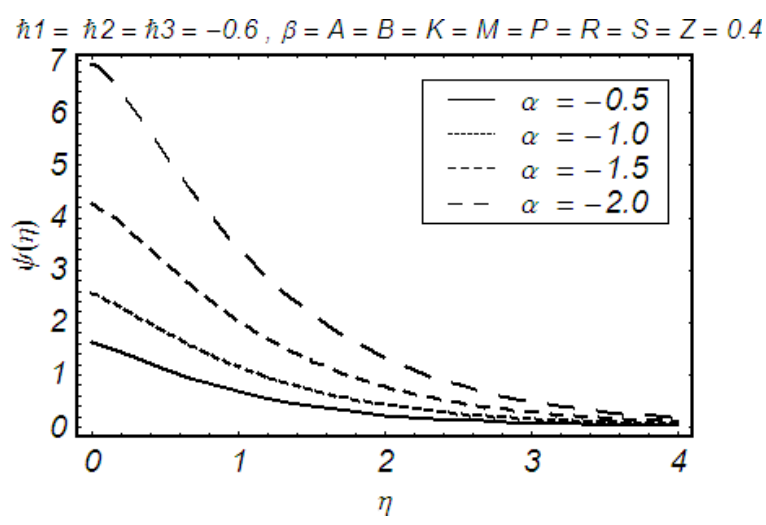

Fig. 62. Influence of shrinking parameter $\alpha$ on $\psi$ for axisymmetric stagnation flow towards a shrinking surface (PHF case).

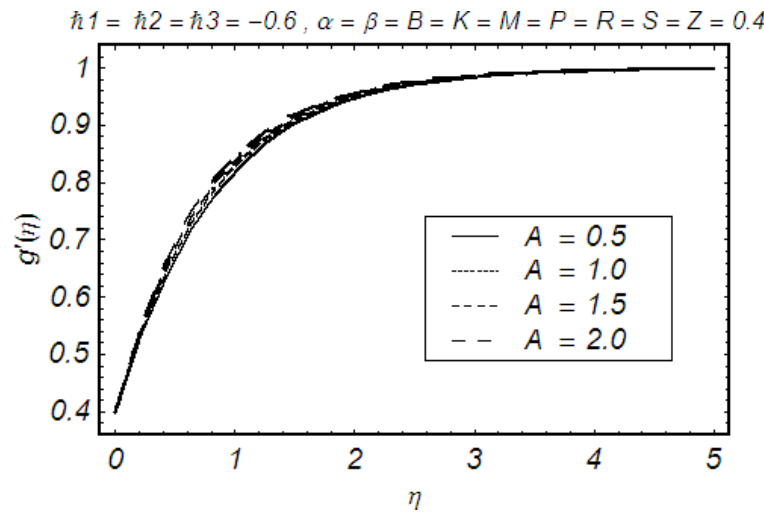

Fig. 63. Influence of non-dimensional parameter parameter $A$ on $g$ for axisymmetric stagnation flow towards a shrinking surface.

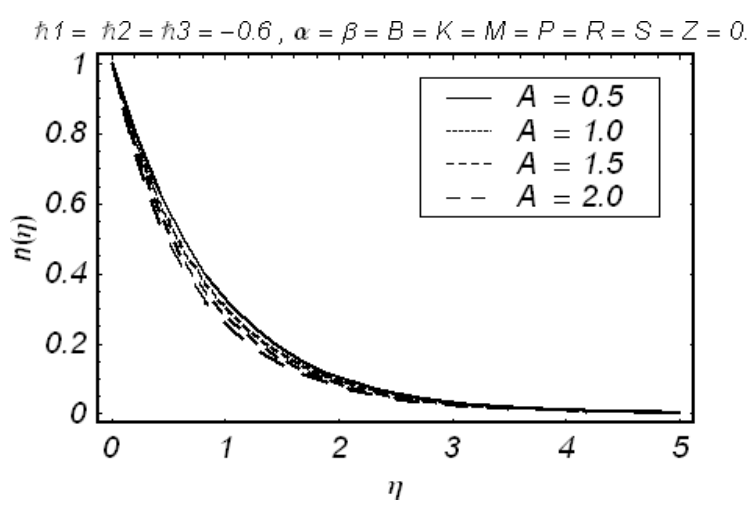

Fig. 64. Influence of non-dimensional parameter parameter $A$ on $n$ for axisymmetric stagnation flow towards a shrinking surface.

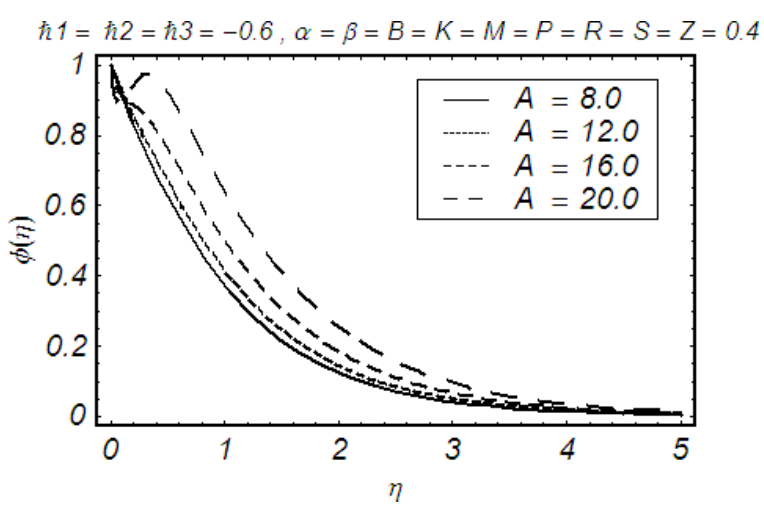

Fig. 65. Influence of non-dimensional parameter parameter $A$ on $\phi$ for axisymmetric stagnation flow towards a shrinking surface (PST case). 


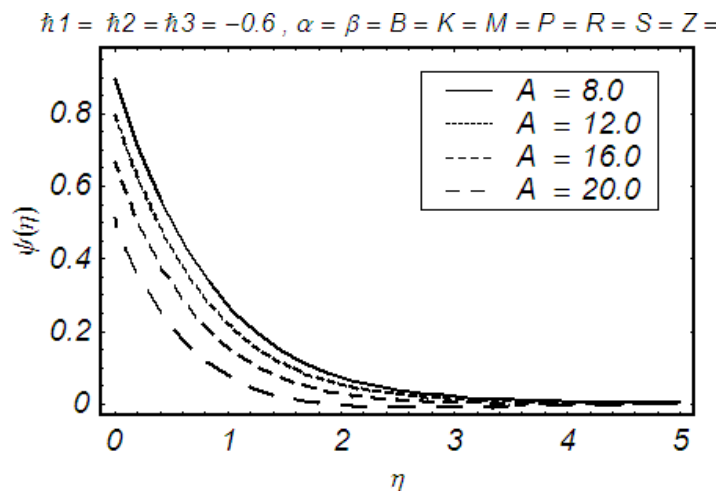

Fig. 66. Influence of non-dimensional parameter parameter $A$ on $\psi$ for axisymmetric stagnation flow towards a shrinking surface (PHF case).

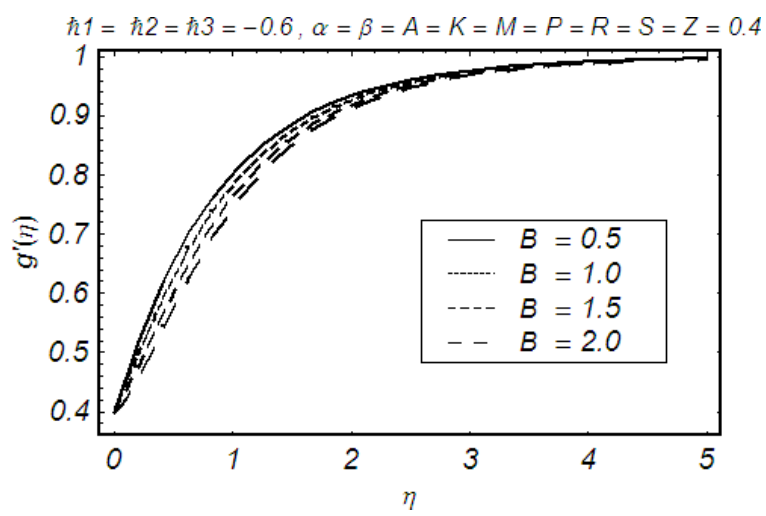

Fig. 67. Influence of non-dimensional parameter parameter $B$ on $g$ for axisymmetric stagnation flow towards a shrinking surface.

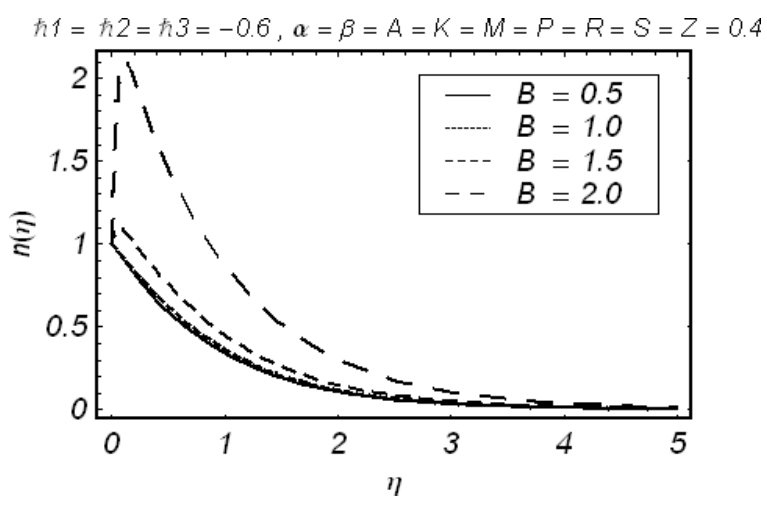

Fig. 68. Influence of non-dimensional parameter parameter $B$ on $n$ for axisymmetric stagnation flow towards a shrinking surface.

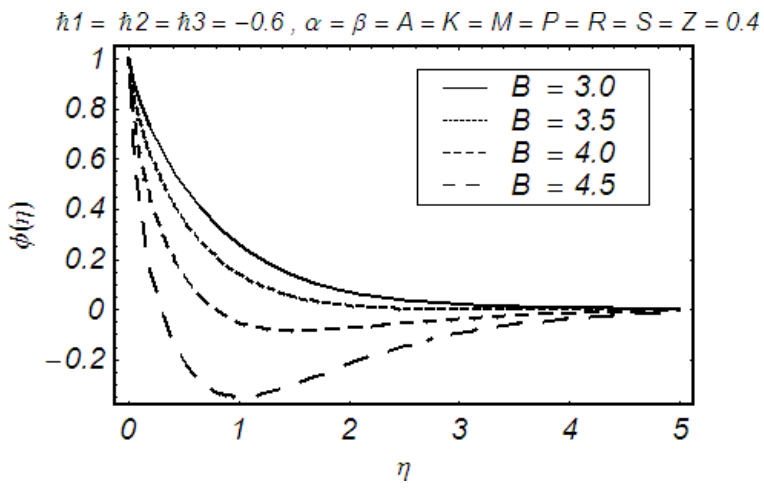

Fig. 69. Influence of non-dimensional parameter parameter $B$ on $\phi$ for axisymmetric stagnation flow towards a shrinking surface (PST case).

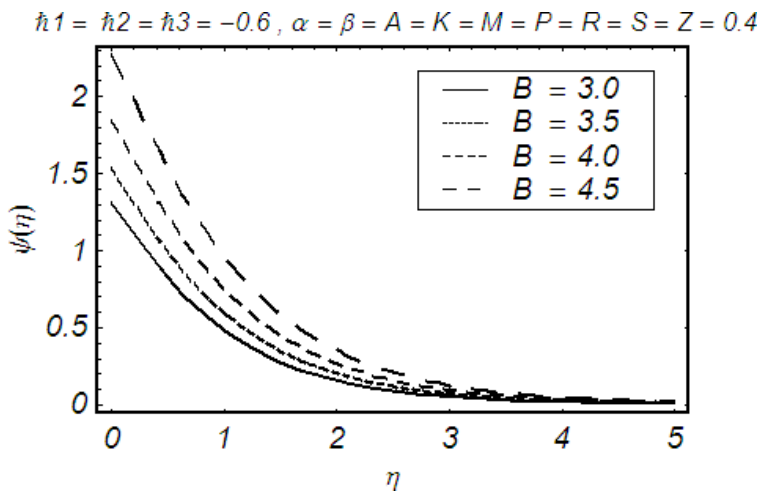

Fig. 70. Influence of non-dimensional parameter parameter $B$ on $\psi$ for axisymmetric stagnation flow towards a shrinking surface (PHF case).

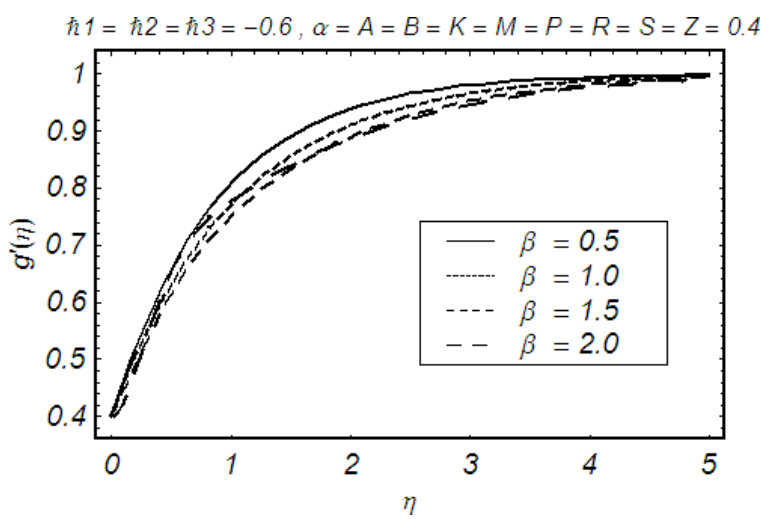

Fig. 71. Influence of non-dimensional parameter parameter $\beta$ on $g$ for axisymmetric stagnation flow towards a shrinking surface. 


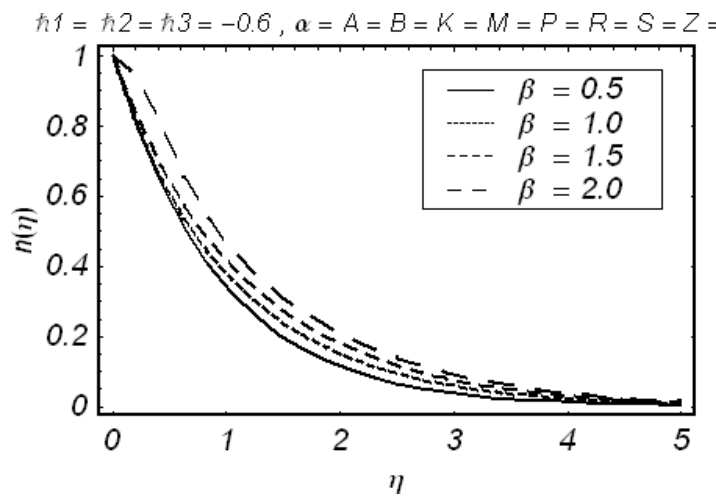

Fig. 72. Influence of non-dimensional parameter parameter $\beta$ on $n$ for axisymmetric stagnation flow towards a shrinking surface.

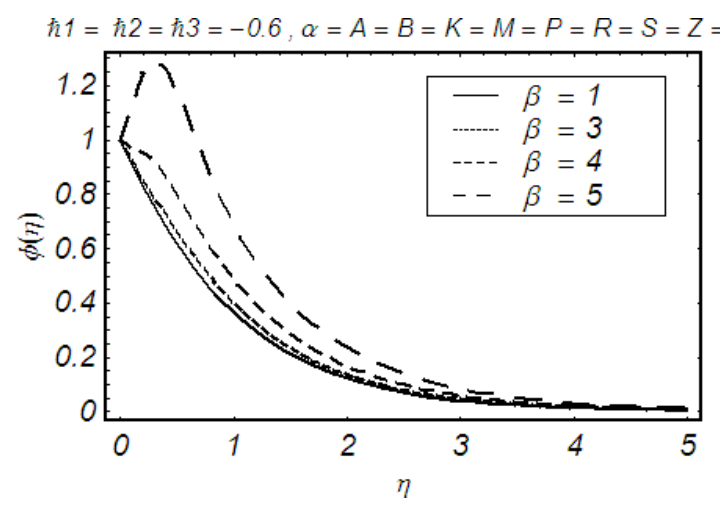

Fig. 73. Influence of non-dimensional parameter parameter $\beta$ on $\phi$ for axisymmetric stagnation flow towards a shrinking surface (PST case).

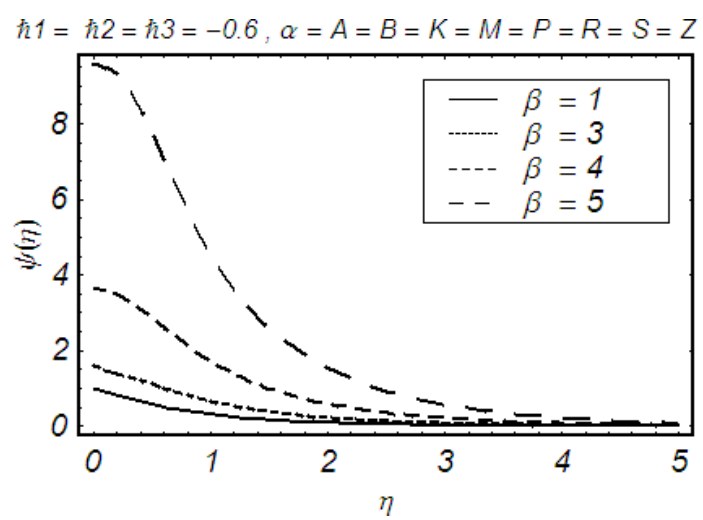

Fig. 74. Influence of non-dimensional parameter parameter $\beta$ on $\psi$ for axisymmetric stagnation flow towards a shrinking surface (PHF case)

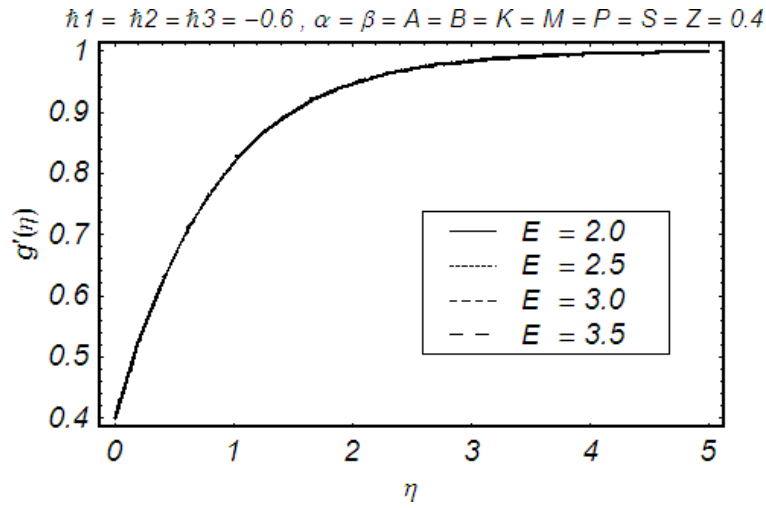

Fig. 75. Influence of Eckert number $E$ on $g$ for axisymmetric stagnation flow towards a shrinking surface.

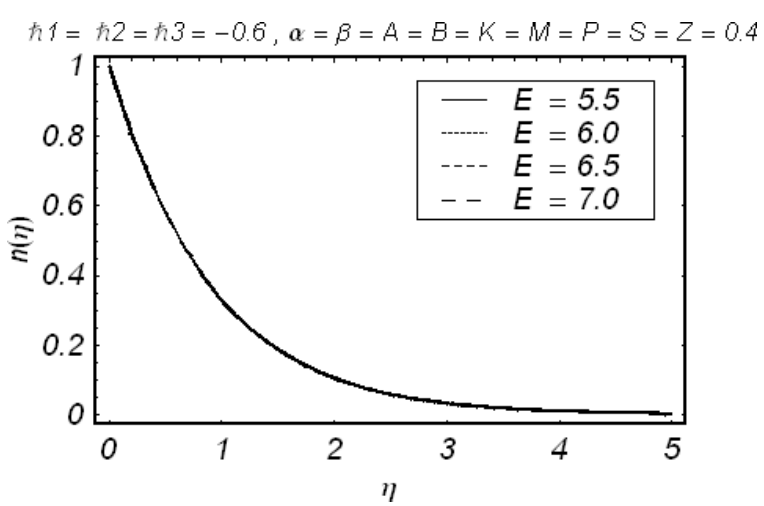

Fig. 76. Influence of Eckert number $E$ on $n$ for axisymmetric stagnation flow towards a shrinking surface.

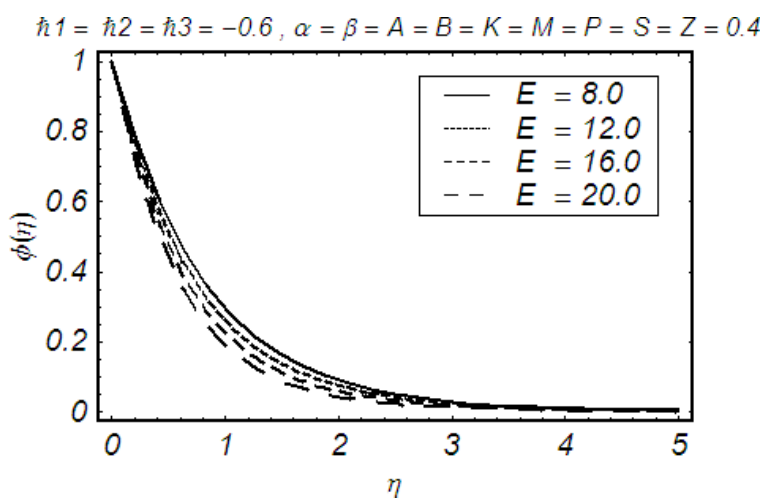

Fig. 77. Influence of Eckert number $E$ on $\phi$ for axisymmetric stagnation flow towards a shrinking surface (PST case). 


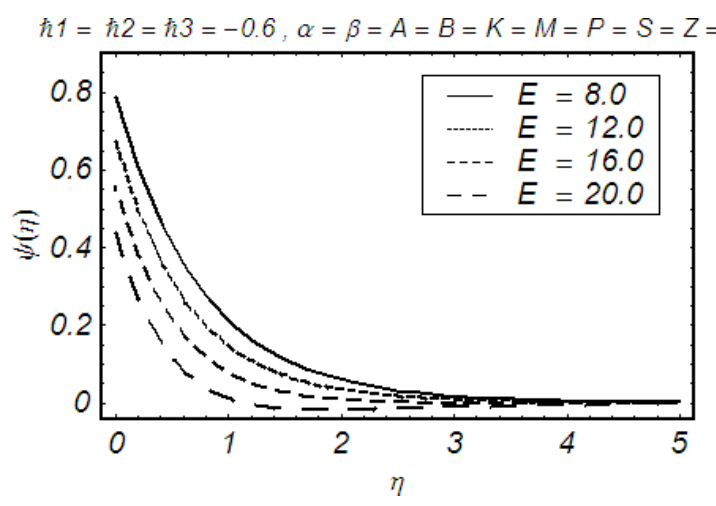

Fig. 78. Influence of Eckert number $E$ on $\psi$ for axisymmetric stagnation flow towards a shrinking surface (PHF case).

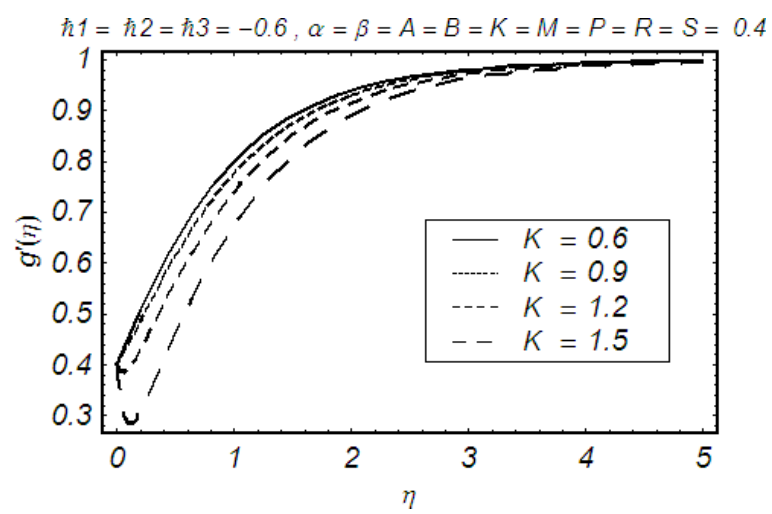

Fig. 79. Influence of non-dimensional parameter parameter $K$ on $g$ for axisymmetric stagnation flow towards a shrinking surface.

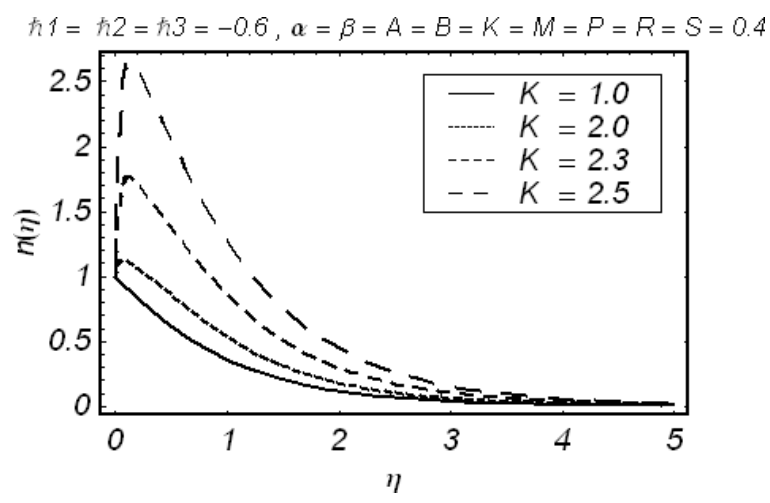

Fig. 80. Influence of non-dimensional parameter parameter $K$ on $n$ for axisymmetric stagnation flow towards a shrinking surface.

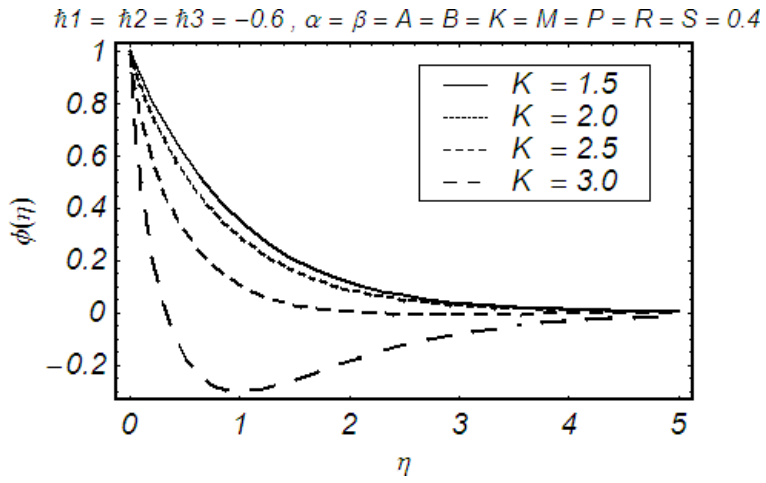

Fig. 81. Influence of non-dimensional parameter parameter $K$ on $\phi$ for axisymmetric stagnation flow towards a shrinking surface (PST case).

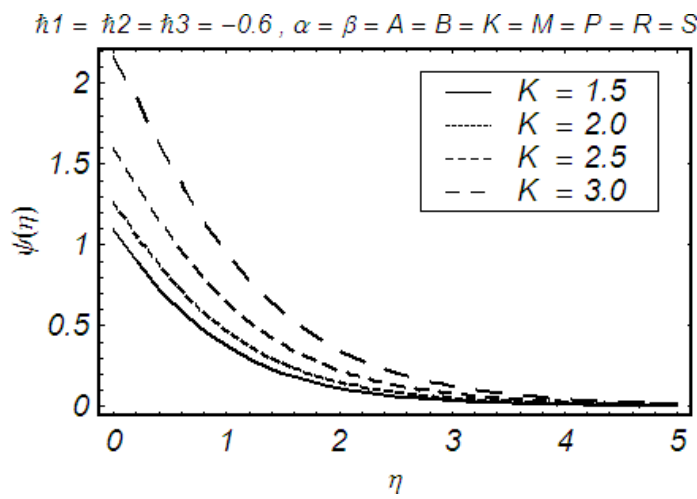

Fig. 82. Influence of non-dimensional parameter parameter $K$ on $\psi$ for axisymmetric stagnation flow towards a shrinking surface (PHF case).

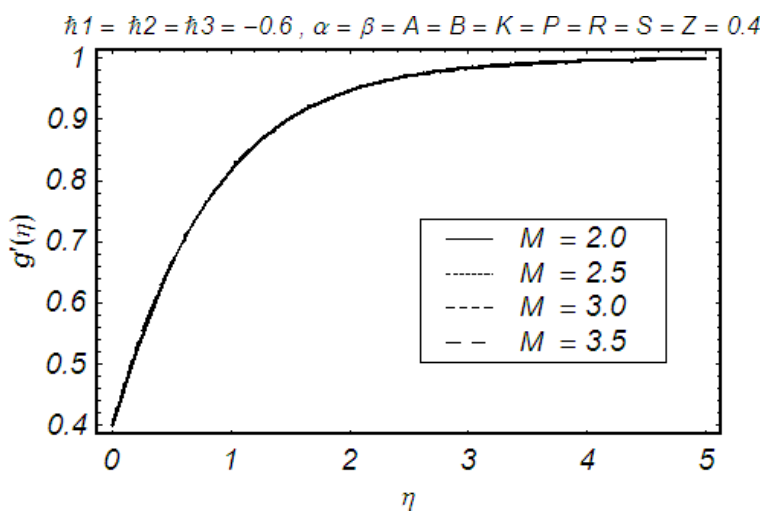

Fig. 83. Influence of non-dimensional parameter parameter $M$ on $g$ for axisymmetric stagnation flow towards a shrinking surface. 


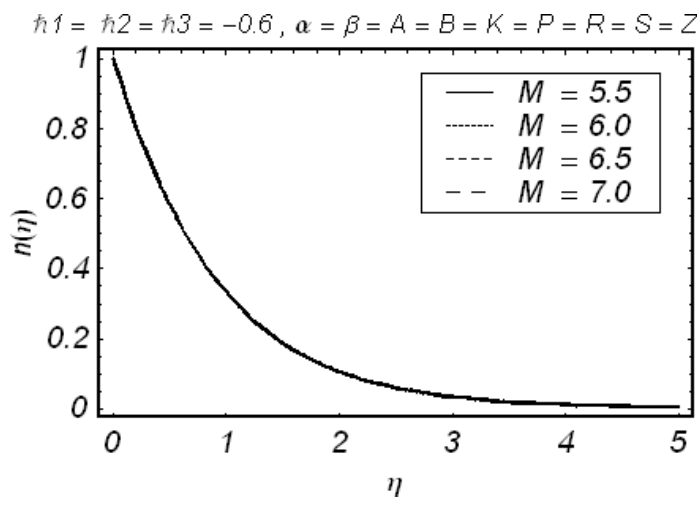

Fig. 84. Influence of non-dimensional parameter parameter $M$ on $n$ for axisymmetric stagnation flow towards a shrinking surface.

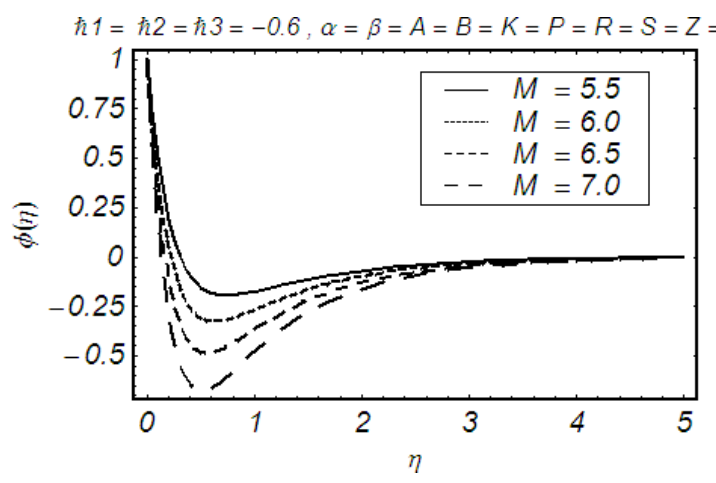

Fig. 85. Influence of non-dimensional parameter parameter $M$ on $\phi$ for axisymmetric stagnation flow towards a shrinking surface (PST case).

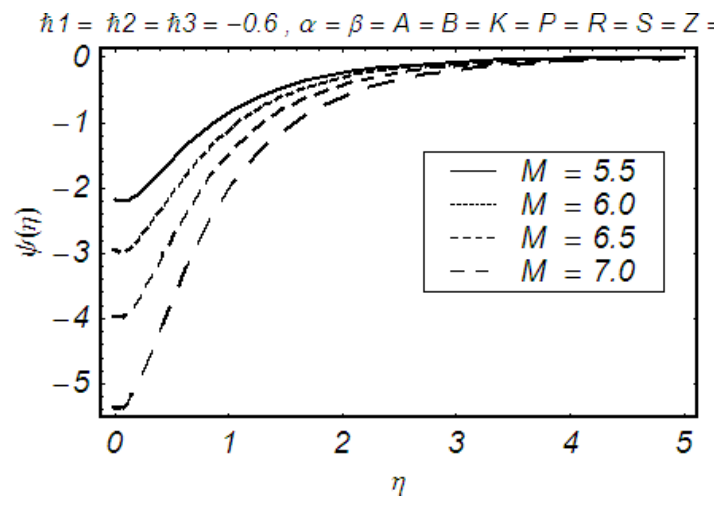

Fig. 86. Influence of non-dimensional parameter parameter $M$ on $\psi$ for axisymmetric stagnation flow towards a shrinking surface (PHF case).

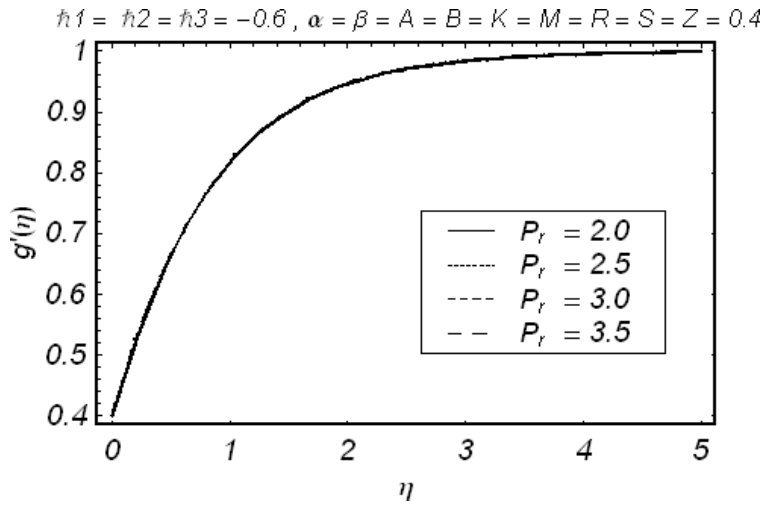

Fig. 87. Influence of Prandtl number $P_{\mathrm{r}}$ on $g$ for axisymmetric stagnation flow towards a shrinking surface.

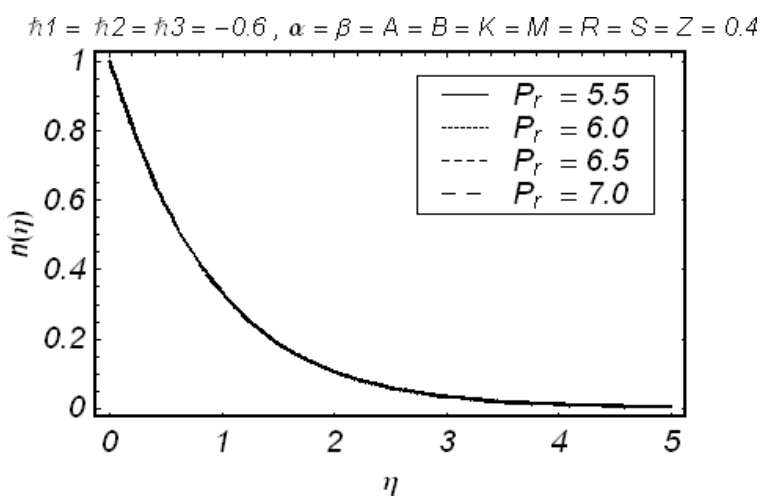

Fig. 88. Influence of Prandtl number $P_{\mathrm{r}}$ on $n$ for axisymmetric stagnation flow towards a shrinking surface.

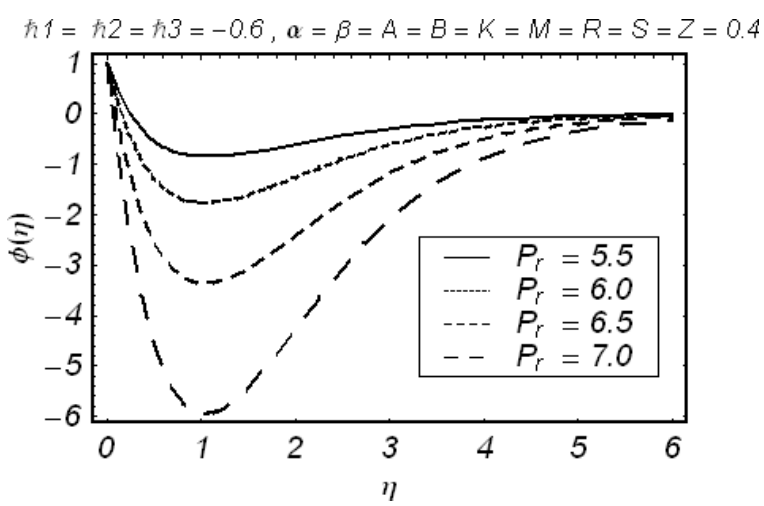

Fig. 89. Influence of Prandtl number $P_{\mathrm{r}}$ on $\phi$ for axisymmetric stagnation flow towards a shrinking surface (PST case). 


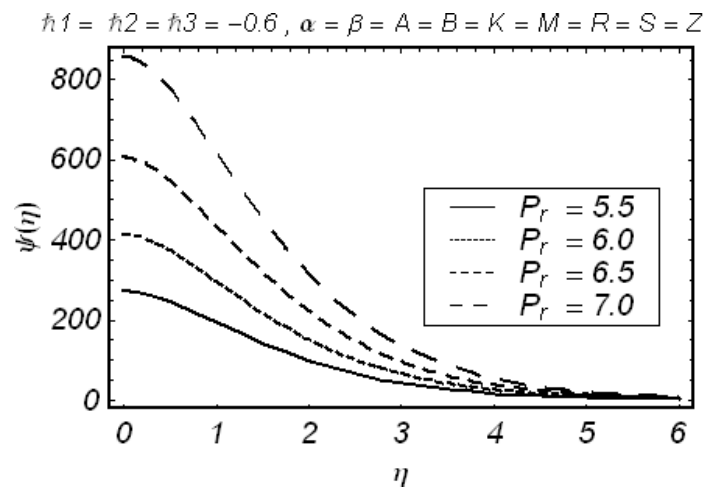

Fig. 90. Influence of Prandtl number $P_{\mathrm{r}}$ on $\psi$ for axisymmetric stagnation flow towards a shrinking surface (PHF case).

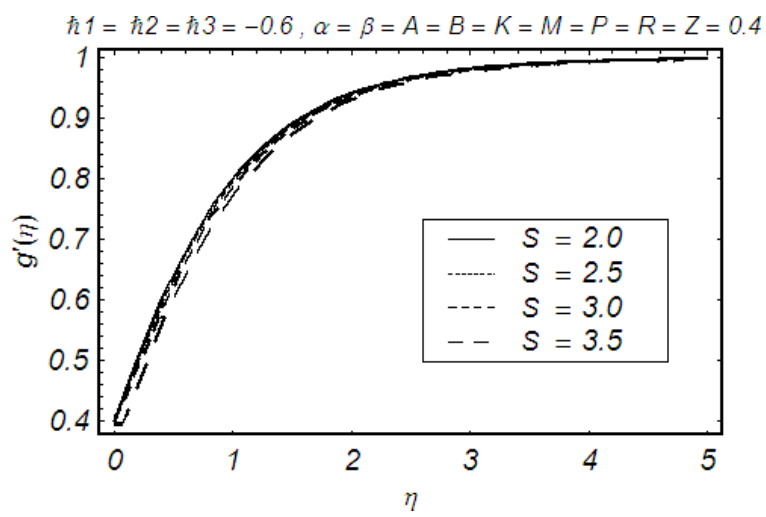

Fig. 91. Influence of non-dimensional parameter $S$ on $g$ for axisymmetric stagnation flow towards a shrinking surface.

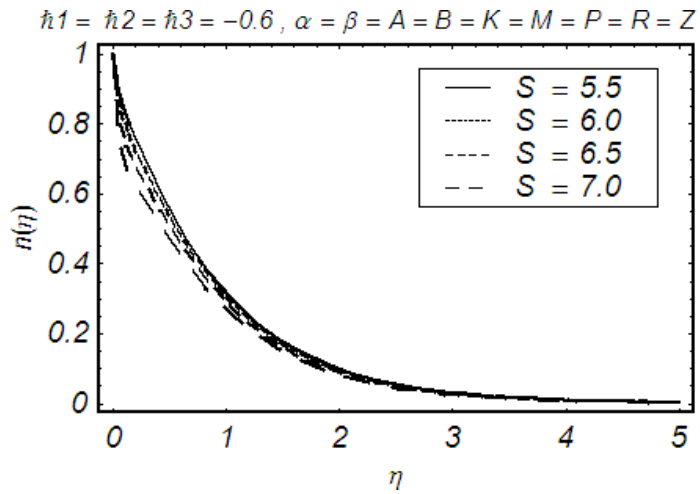

Fig. 92. Influence of non-dimensional parameter $S$ on $n$ for axisymmetric stagnation flow towards a shrinking surface.

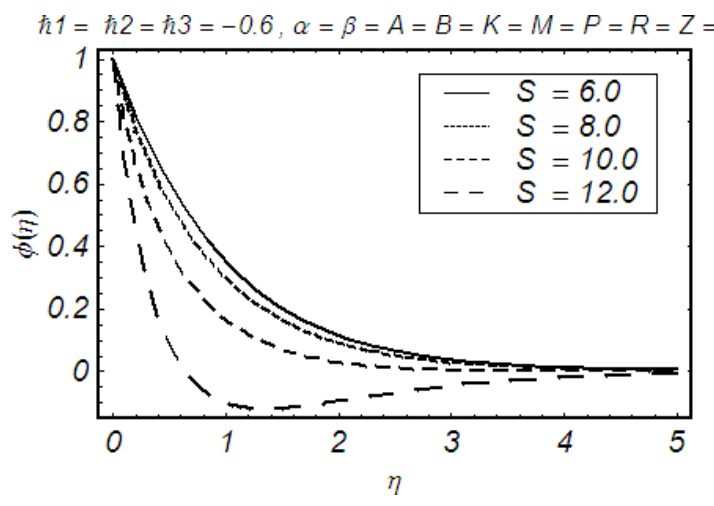

Fig. 93. Influence of non-dimensional parameter $S$ on $\phi$ for axisymmetric stagnation flow towards a shrinking surface (PST case).

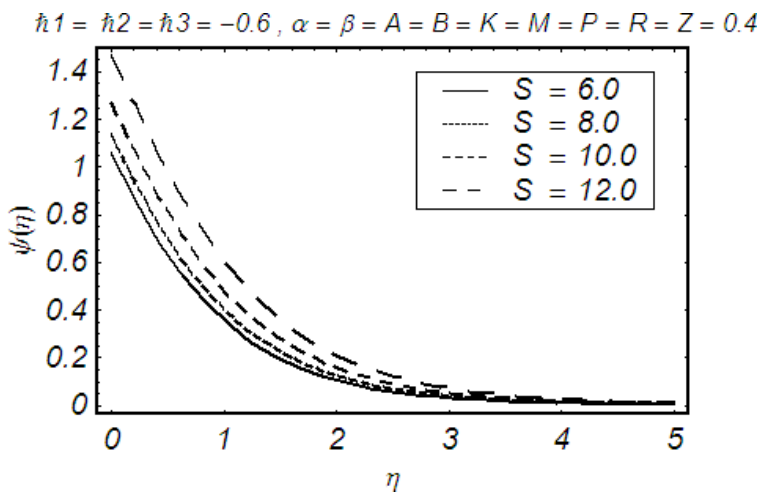

Fig. 94. Influence of non-dimensional parameter $S$ on $\psi$ for axisymmetric stagnation flow towards a shrinking surface (PHF case).

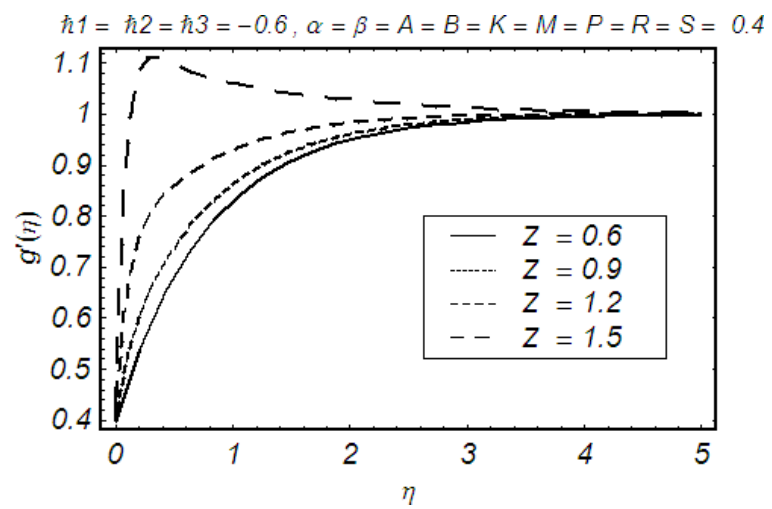

Fig. 95. Influence of non-dimensional parameter $Z$ on $g$ for axisymmetric stagnation flow towards a shrinking surface. 


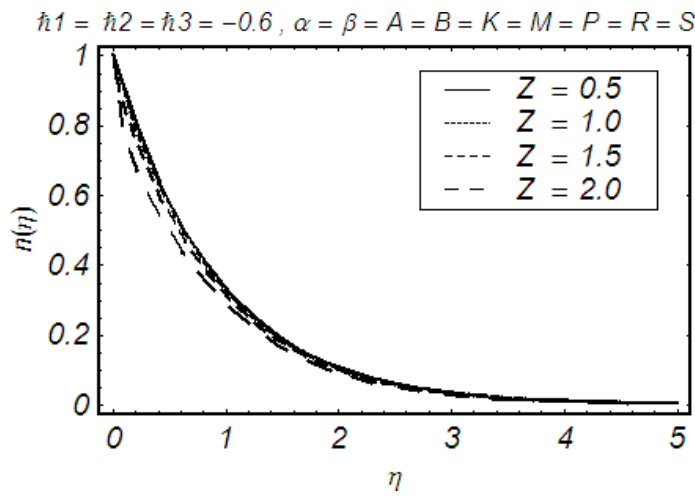

Fig. 96. Influence of non-dimensional parameter $Z$ on $n$ for axisymmetric stagnation flow towards a shrinking surface.

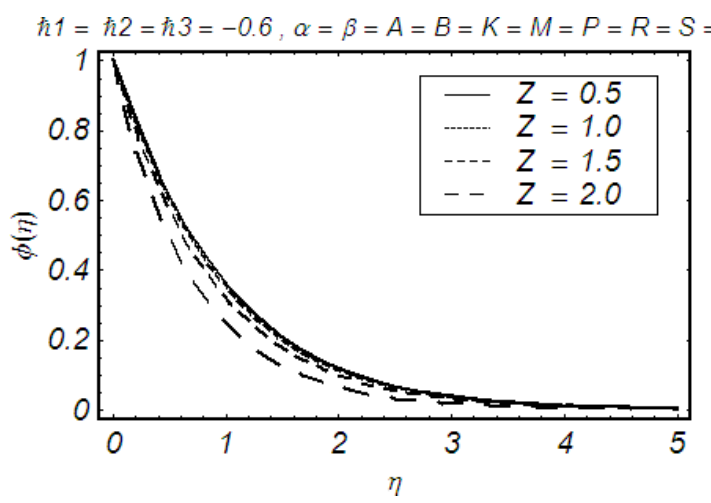

Fig. 97. Influence of non-dimensional parameter $Z$ on $\phi$ for axisymmetric stagnation flow towards a shrinking surface (PST case).

[1] B. C. Sakiadis, AIChE J. 7, 26 (1961).

[2] H. Xu, S. J. Liao, and I. Pop, Europ. J. Mec. B/Fluids 26, 15 (2007).

[3] S. J. Liao, Commun. Nonlinear Sci. Numer. Simul. 11, 326 (2006).

[4] S. J. Liao, Int. J. Heat Mass Trans. 48, 2529 (2005).

[5] M. Sajid and T. Hayat, Int. Commun. Heat Mass Transf. 35, 347 (2008).

[6] M. A. El-Aziz, Meccan. 42, 375 (2007). DOI10.1007 / s11012-006-9051-5.

[7] M. Sajid and T. Hayat, I. Commun. Heat Mass Transf. 35, 347 (2008).

[8] I. C. Liu and H. I. Andersson, Int. J. Therm. Sci. 47, 766 (2008).

[9] S. K. Khan and E. Sanjayanand, Int. J. Heat Mass Transf. 48, 1534 (2005).

[10] E. M. A. Elbashbeshy and M. A. A. Bazid, Appl. Math. Comput. 138, 239 (2003).

[11] E. Magyari and B. Keller, J. Phys. D: Appl. Phys. 32, 577 (199).

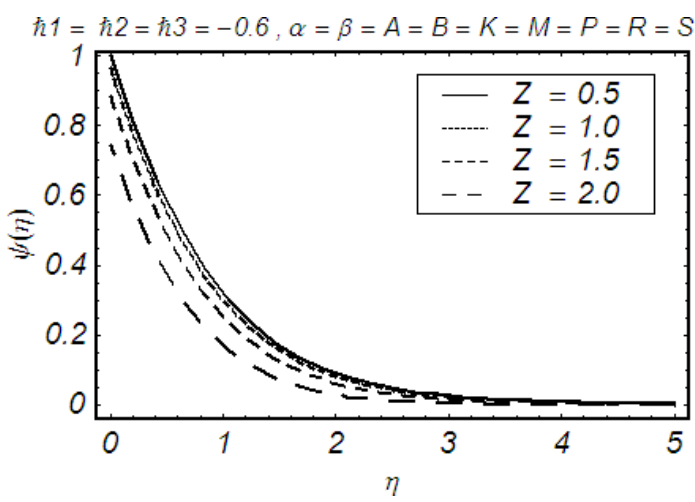

Fig. 98. Influence of non-dimensional parameter $Z$ on $\psi$ for axisymmetric stagnation flow towards a shrinking surface (PST case).

parameter $Z$. Figures $75-76$ reflect no change in $g^{\prime}$, $n$ and Figures 77-78 indicate a decrease in $\phi$ and $\psi$ with an increase in the Eckert number $E$. The results are similar for the non-dimensional parameter $M$. These are shown in Figures 83-86. No change has been found in $g^{\prime}, n$; while $\phi$ and $\psi$ show an opposite behaviour with an increase in the Prandtl number $P_{\mathrm{r}}$ (see Figs. 87-90).

[12] M. Zakaria, Appl. Math. Comput. 155, 165 (2004).

[13] E. M. A. Elpashbeshy and M. A. A. Bazid, Appl. Math. Comput. 158, 799 (2004).

[14] T. R. Mahapatra and A. S. Gupta, Acta Mech. 152, 191 (2001).

[15] R. Nazar, N. Amin, D. Filip, and I. Pop, Int. J. Eng. Sci. 42, 1241 (2004).

[16] Y. Y. Lok, N. Amin, and I. Pop, Int. J. Therm. Sci. 45, 1149 (2006).

[17] L. Y. Yian, N. Amin, and I. Pop, Int. J. Heat Mass Transf. 50, 4855 (2007).

[18] T. Hayat, Z. Abbas, T. Javed, and M. Sajid, Chaos, Solitons, and Fractals 39, 1615 (2009).

[19] S. Nadeem and M. Awais, Phys. Lett. A 372, 4965 (2008).

[20] T. Fang, Heat Mass Transf. (2008), doi:10.1016 / j.ijheatmasstransfer.2008.04.067.

[21] T. Hayat, T. Javed, and M. Sajid, Phys. Lett. A 372, 3264 (2008). 
[22] T. Hayat, Z. Abbas, and N. Ali, Phys. Lett. A 372, 4698 (2008).

[23] C. Y. Wang, Int. J. Nonlinear Mech. 43, 377 (2008).

[24] M. Sajid and T. Hayat, Appl. Math. Comput. 189, 1576 (2007).

[25] M. Sajid, T. Hayat, and S. Asghar, Int. J. Heat Mass Transf. 50, 1723 (2007).

[26] S. J. Liao, Beyond perturbation introduction to homotopy analysis method, Chapman \& Hall/CRC Press, Boca Raton 2003.

[27] H. Xu, S. J. Liao, and X. C. You, Commun. Nonlinear Sci. Numer. Simul. 14, 1152 (2009).

[28] S. J. Liao, Commun. Nonlinear Sci. Numer. Simul. 14, 983 (2009).
[29] S. J. Liao, Nonlinear Analysis: Real World Appl. 10, 2455 (2009).

[30] S. Abbasbandy and T. Hayat, Commun. Nonlinear Sci. Numer. Simul. (2009), doi:10.1016 / j.cnsns.2009.01.030.

[31] S. Abbasbandy, Chaos, Solitons, and Fractals, 39, 428 (2009).

[32] S. Nadeem, T. Hayat, S. Abbasbandy, and M. Ali, Nonlinear Analysis: Real World Appl. (2009), doi:10.1016 / j.nonrwa.2009.01.030.

[33] M. S.H. Chowdhury, I. Hashim, and O. Abdulaziz, Nonlinear Sci. Numer. Simul. 14, 371 (2009).

[34] A. S. Bataineh, M. S. M. Noorani, and I. Hashim, Commun. Nonlinear Sci. Numer. Simul. 14, 409 (2009). 